regulator problem; the algebraic Riccati equation," to appear in SIAM J. Contr.

[31] F. J. Helton and J. W. Helton, "Scattering theory for computers and other nonlinear systems," to appear.

[32] J. W. Helton and J. V. Ralston, "The first variation of the scattering matrix," to appear.

[33] E. Hille and R. S. Phillips, "Functional analysis and semigroups," AMS Colloq., vol. 31.

[34] E. Kamen, "On an algebraic theory of systems defined by convolution operators," to appear in Math Syst. Theory.

[35] - ", "Topological module structure of linear continuous time systems."

[36] -, "Applications of modern algebra to infinite dimensional systems."

[37] P. D. Lax and R. S. Phillips, Scattering Theory. New York: Academic Press, 1967.

[38] -, "Scattering theory for dissipative hyperbolic systems," $J$. Funct. Anal., vol. 14, pp. 172-235, 1973.

[39] -, "Decaying modes for the wave equation in the exterior of an obstacle," Commun. Pure Appl. Math., vol. XXVII, pp. 737$787,1969$.

[40] -, "A logrithmic bound on the location of the poles of the scattering matrix," Arch. Inst. Mech. Anal., vol. 40, no. 4, pp. 268$280,1971$.

[41] -, "Scattering theory for acoustic equation in an even number of space dimensions," Indiana J., vol. 22, pp. 101-134, 1972.

[42] - "On the scattering frequencies of the Laplace operator for exterior domains."

[43] J. L. Lions, Optimal Control of Systems Governed by Partial Differential Equations. New York: Springer, 1971.

[44] M. S. Livsic, "Operators oscillations, waves, open systems," AMS Trans. Monogr., vol. 34, 1973.
[45] -, "Theory of operator nodes on Hilbert spaces," 1971, in Russian.

[46] A. Majda, "Disappearing solutions for the dissipative wave equation, to appear.

[47] C. Morawetz, "Decay for solutions of Dirchlet exterior problem for the wave equation."

[48] C. Morawetz and D. Ludwig.

[49] B. Sz-Nagy and C. Foias, Harmonic Analysis of Operators on Hilbert Space. Amsterdam, The Netherlands: North Holland, 1970.

[50] J. V. Ralston, "Solutions of the wave equation with localized energy," Commun. Pure Appl. Math., vol. XXII, pp. 807-823, 1969.

[51] _, "Trapped rays in spherically symmetric media and poles of the scattering matrix," Commun. Pure Appl. Math., vol. XXIV, pp. 571-582, 1971 .

[52] -, "Variation of the transmission coefficient and comparison theorems for the purely imaginary polar of the scattering matrix," Commun. Pure Appl. Math., vol. XXV, pp. 45-61, 1972.

[53] —, "Local decay of solutions of conservative first order hyper. bolic systems in odd dimensional space," Trans. $A M S$, vol. 194, 1974.

[54] -, "On the propagation of singularities in solutions of sym metric hyperbolic partial differential equations."

[55] M. Reed and B. Simon, Methods of Modern Mathematics and Physics: Functional Analysis. New York: Academic Press, 1972.

[56] F. Riesz and B. Sz. Nagy, Functional A nalysis. New York: Ungar, 1955.

[57] W. Rudin, Functional Analysis. New York: McGraw-Hill, 1973.

[58] W. Strauss, "Dispersion of waves vanishing on the boundary of an exterior domain," to appear.

[59] Yosida, Functional Analysis. New York: Springer, 1966.

\title{
Invariant Subspace Methods in Linear Multivariable-Distributed Systems and Lumped- Distributed Network Synthesis
}

\author{
JOHN S. BARAS, MEMBER, IEEE, AND PATRICK DEWILDE, MEMBER, IEEE
}

\begin{abstract}
Linear multivariable-distributed systems and synthesis problems for lumped-distributed networks are analyzed. The methods used center around the invariant subspace theory of Helson-Lax and the theory of vectorial Hardy functions. Statespace and transfer function modek are studied and their relations analyzed. We single out a class of systems and networks with nonrational transfer functions (scattering matrices), for which several of the well-known results for lumped systems and networks are generalized. In particular we develop the relations between singubarities of transfer functions and "natural modes" of the systems, a degree theory for infinite-dimensional linear systems and a synthesis via lossless embedding of the scattering matrix. Finally coprime factorizations for this class of systems are developed.
\end{abstract}

Manuscript received April 1, 1975 ; revised August 1, 1975. The work of J.S. Baras was supported in part by a General Research Board Award from the University of Maryland and the work of $P$. Dewilde was supported in part by the Belgian National Fund for Scientific Research (NFWO).

J. S. Baras is with the Electrical Engineering Department, University of Maryland, College Park, MD 20742.

P. Dewilde is with the Departement Elektrotechniek, Katholieke Universiteit Leuven, Heverlee, Belgium.
These factorizations play an essential role in the development and show that properties of Hardy functions are of fundamental importance for this class of distributed systems as properties of rational functions ate for lumped systems.

\section{INTRODUCTION}

\4 REQUENCY DOMAIN methods in 'nmped-multivariable systems have been developed in the last few years for the analysis and design of control systems [1]. These methods provided a clear understanding of the interrelations between state-space and transfer function models for systems and networks and proved to be extremely useful in practical design applications [82].

Recently, several researchers, Baras [2]-[8], Brockett [3][4], Dewilde [9]-[16], Fuhrmann [17]-[23], Helton [24] and [25], have been investigating a similar approach to the analysis and synthesis of distributed systems and networks. This theory applies to situations where energy considerations provide the setting of a Hilbert space for the state space of the 
distributed system or network considered. The major mathematical tools are the invariant subspace theory of Helson [26], Beurling [27], Lax [28], the associated theory of Hardy spaces of analytic functions Hoffman [29], Düren [30] and general harmonic analysis of operators and semigroups in Hilbert spaces of Nagy and Foias [31]. The results obtained thus far include a fairly complete parallel development of state-space and transfer function models for systems and networks and important synthesis methods. The foundations for the development of a detailed theory, as in the lumped case, have been laid and the subject is growing considerably.

One of the most valuable advantages of these new investigations, is their potential use in the development of practical approximation schemes, in the design of suboptimal contollers and filters, and in the synthesis of distributed systems and networks. Although these research efforts started relatively scently, we would like to point out that the Russian literature contains many examples of investigation along similar lines (i.e., relations between operator theory-system theory-network theory) [32]-[37], as illustrated by the recent translation of the book by Livšic [37].

The present paper is an outgrowth of research on the subject by the authors and includes both new results and short summaries of known results. It is structured as follows: Section I contains some necessary mathematical background and notations. Section II develops the state-space and frequencydomain theory of linear multivariable distributed systems and is divided in four subsections: one describing state-space models; one devoted to transfer function models; one describing input-output properties and a module theoretic setting, and one analyzing the relations between singularities of transfer functions and spectral properties of the corresponding operators in the space model (this part is primarily written by J.S. varas). Section III develops state-space and frequency-domain synthesis methods for lumped-distributed networks and is also divided in four subsections; one describing the network under consideration; one providing the connections with Section II and the state-space theory of scattering matrices; one developing a scattering matrix synthesis through lossless embedding and one analyzing transfer scattering matrix synthesis (this part is primarily written by $P$. Dewilde). Finally Section IV contains the conclusions and some suggestions for further research.

\section{Mathematical Preliminaries}

The following spaces will be used in the paper:

$C^{n} \quad$ The Euclidean space of complex

$L^{p}[a, b](1 \leqslant p \leqslant \infty)$ The well-known Lebesgue spaces.

$L_{n}^{p}[a, b](1 \leqslant p \leqslant \infty)$ The, standard vector-valued (with values in $\mathbb{C}^{n}$ ) Lebesgue spaces [30].

$H^{p}(1 \leqslant p<\infty) \quad$ The space of functions $f$ analytic in the open right-half complex plane (ORP), such that

$$
\sup _{\sigma>0}\|f(\sigma+j \omega)\|_{L} \leqslant M<\infty .
$$

$H^{\infty} \quad$ The space of functions $f$, analytic and bounded in ORP. $H^{\infty}$ is clearly an algebra [29].

$H_{n}^{p}(1 \leqslant p<\infty) \quad$ The space of vector valued functions (with values in $\boldsymbol{C}^{n}$ ) with every component being an element of $H^{p}$

In the above spaces the measure used on the imaginary axis is $d \omega / 2 \pi$.

$$
\begin{aligned}
& H_{m \times n}^{\infty} \\
& d^{H^{\infty}}, d^{H_{n}^{p}}, d^{H_{m \times n}^{\infty} \times}
\end{aligned}
$$

The space of $m \times n$ matrices with elements in $H^{\infty}$.

Same as above with ORP replaced by the open unit disk.

Let $N$ be a Hilbert space and $\mathcal{L}\left(\mathbb{C}^{n}, N\right)$ the Hilbert space of continuous linear operators from $\mathbb{C}^{n}$ into $N$ with inner product

$$
\left\langle B_{1}, B_{2}\right\rangle=\operatorname{tr}\left(B_{1}^{*} B_{2}\right)
$$

$H^{2}\left(\mathcal{L}\left(\mathbb{C}^{n}, N\right)\right)$ is the space of $\mathcal{L}\left(\mathbb{C}^{n}, N\right)$-valued analy tic functions $F$ in ORP such that

$$
\sup _{\sigma>0} \frac{1}{2 \pi} \int_{-\infty}^{\infty}\|F(\sigma+j \omega)\|_{\mathcal{L}\left(\mathcal{C}^{n}, N\right)}^{2} d \omega \leqslant M<\infty .
$$

The theory of the Hardy spaces $H^{p}, H_{n}^{p}, H_{m \times n}^{\infty}$ is well documented [26], [29], [30] but we summarize here the results which we will use in the sequel. First, it is known that functions belonging to a Hardy space have nontangential limits almost everywhere (a.e.) on the imaginary axis. For a function in $H_{n}^{2}$, this limit belongs to $L_{n}^{2}(-j \infty, j \infty)$, and for a function in $H_{m \times n}^{\infty}$ the limit belongs to $L_{m \times n}^{\infty}(-j \infty, j \infty)$ and we will assume the function defined on the closed right-half plane. It is known (a convexity argument) that functions in $H_{n}^{p}(1 \leqslant p \leqslant \infty)$ attain their norm on the boundary so that there is a Banach space isomorphism between the boundary functions and the Hardy space. Hence $H_{n}^{p}$ can be viewed as a subspace of $L_{n}^{p}(-j \infty, j \infty), H_{m \times n}^{\infty}$ as a subspace of $L_{m \times n}^{\infty}$ : namely that subspace whose elements have an appropriate analytic extension to the ORP. Often we will call a function $F \in L_{n}^{p}(-j \infty, j \infty)$ or $L_{m \times n}^{\infty}(-j \infty, j \infty)$ analytic if $F$ is the boundary value of an $H_{n}^{p}$ function or $H_{m \times n}^{\infty}$ function. It will be called conjugate analytic if it has a similar extension to the open left-half complex plane (OLP). Only constant functions can be both analytic and conjugate analytic. Let $\mathcal{F}$ be the Fourier map:

$$
\mathcal{F}: L_{n}^{2}(-\infty, \infty) \longmapsto L_{n}^{2}(-j \infty, j \infty) ; f(t) \longmapsto \int_{-\infty}^{\infty} f(t) e^{-j \omega t} d t
$$

$\mathcal{F}$ is a Hilbert space isomorphism (Plancherel's theorem) and $\mathfrak{F}^{-1}$ is given by

$\mathcal{F}^{-1}: L_{n}^{2}(-j \infty, j \infty) \longrightarrow L_{n}^{2}(-\infty, \infty)$

$$
F(j \omega) \longrightarrow \int_{-\infty}^{\infty} F(j \omega) e^{j \omega t} \frac{d \omega}{2 \pi} .
$$

The Paley-Wiener theorem asserts that $H_{n}^{2}=\mathfrak{F} L_{n}^{2}(0, \infty)$, so that analytic functions in $L_{n}^{2}(-j \infty, j \infty)$ are precisely transforms of functions in $L^{2}(-\infty, \infty)$ which are zero for $t<0$. Let $K_{n}^{2}$. be the Hilbert space of conjugate analytic functions in $L_{n}^{2}(-j \infty$, $j \infty)$, then $L_{n}^{2}(-j \infty, j \infty)=K_{n}^{2} \oplus H_{n}^{2}$ (direct sum of orthogonal subspaces) since $L_{n}^{2}(-\infty, \infty)=L_{n}^{2}(-\infty, 0) \oplus L_{n}^{2}(0, \infty)$.

In $L_{n}^{2}(-\infty, \infty)$ or $L_{n}^{2}(-j \infty, j \infty)$ we will use the following operators:

$\sigma_{\tau}$ left shift by $\tau: \sigma_{\tau} f(t)=f(t+\tau)$

$\sigma_{-}$argument sign reversal $: \sigma_{-} f(t)=f(-t)$ or $\sigma_{-} F(j \omega)=$ $F(-j \omega)$.

For $g$ in $L_{n}^{2}(-\infty, \infty)$, we have that $\mathcal{F} \sigma_{\tau} g=e^{j \omega \tau} \mathcal{F} g$ and that $\mathcal{F}_{\sigma_{-}} \boldsymbol{g}$ is conjugate analytic if $\mathcal{F} g$ is analytic. A linear map $f: L_{n}^{2}(-\infty, \infty) \rightarrow L_{m}^{2}(-\infty, \infty)$ is said to be translation invariant if $f \sigma_{\tau}=\sigma_{\tau} f$ for all $\tau$. Suppose $f$ is a linear bounded and translation invariant map from $L_{n}^{2}(-\infty, \infty)$ into $L_{m}^{2}(-\infty, \infty)$. The 
Bochner-Chandrasekharan theorem [38] asserts (with an extension to the vector situation) that $f$ can be represented multiplicatively in the transform domain:

Namely if $b(t)=f a(t)$ and $A=\mathfrak{F} a, B=\mathfrak{F} b$, then $B(j \omega)=$ $S(j \omega) A(j \omega)$ where $S(j \omega)$ is an $m \times n$ matrix, $S \in L_{m \times n}^{\infty}(-j \infty$, $j \infty)$ and the norm of $f$ equals $\|S\| L_{m}^{\infty} \times n$.

If moreover $f\left\{L_{n}^{2}(0, \infty)\right\} \subset L_{m}^{2}(0, \infty)$, then $S$ is analytic and has an extension to the ORP, as described earlier. In case $f$ is contractive (i.e., has norm $\leqslant 1$ ), then $1_{n}-\tilde{S}(j \omega) S(j \omega) \geqslant$ 0 a.e. $\omega$. $(\tilde{S}(j \omega)$ is the Hermitian conjugate of $S(j \omega))$.

$\tilde{S}(j \omega)$ is conjugate analytic if $S(j \omega)$ is analytic. An analytic matrix function with unitary values on the imaginary axis is called an inner function. A function $\varphi \in H^{\infty}$ is inner if $|\varphi(j \omega)|=1$ a.e. $\omega$ (e.g., $\left.(p-1) /(p+1), e^{-p}\right)$. A subspace h $\subset H_{n}^{2}$ will be called an invariant subspace if it is closed and $G$ 尓 $\subset$ for all $G \in H^{\infty}$. Because of an approximation theorem we will have that is an invariant subspace if and only if either

$$
\left(\frac{j \omega-1}{j \omega+1}\right)^{n} \pi \subset \text { for all } n=0,1,2, \cdots
$$

or $e^{-j \omega t} \backslash \subset$ for all $t \geqslant 0$. The Beurling-Lax theorem asserts that any invariant subspace $\subset H_{n}^{2}$ has the form $=U \cdot H_{k}^{2}$ where $U \in H_{n \times k}^{\infty}, k \leqslant n$, and $U(j \omega)$ is an isometry a.e. $\omega$. We will say that has full range if $k=n$. In the latter case, functions in $\operatorname{span} \mathbb{C}^{n}$ a.e. $\omega$ and $U(j \omega)$ is unitary. A matrix $A \in H_{k \times n}^{\infty}$ such that $\overline{A H_{n}^{2}}=H_{k}^{2}$ (where the overbar indicates closure) is called outer. Any function $g \in H^{\infty}$ has a factorization $g=\varphi g_{1}$ with $\varphi$ inner and $g_{1}$ outer. These functions can be factored further and we have that [29]

$$
\varphi(p)=c_{0} b(p) \cdot s(p)
$$

with $c_{0}$ a constant, $b$ a Blaschke product containing all the zeros $\left\{p_{i}\right\}$ of $\varphi$ in ORP ( $\tilde{p}_{i}$ is the complex conjugate of $p_{i}$ ), and $s$ a singular part:

$b(p)=\left(\frac{p-1}{p+1}\right)^{k} \prod_{i} \frac{p-p_{i}}{p+\tilde{p}_{i}} \cdot \frac{\left|1-p_{i}^{2}\right|}{1-p_{i}^{2}}, \quad$ where $\sum_{i} \frac{\operatorname{Re} p_{i}}{1+\left|p_{i}^{2}\right|}<\infty$ $s(p)=e^{-p r} \exp \left[-\int_{-\infty}^{\infty} \frac{t p+j}{t+j p} d \mu(t)\right]$

where $r>0$, and $\mu$ is a finite singular positive measure on the $j \omega$ axis. Notice that inner (respectively outer) functions are generalizations of the all-pass functions, respectively minimal phase functions, of the lumped-network theory.

Two functions $F_{1} \in H_{m \times k}^{\infty}, F_{2} \in H_{m \times l}^{\infty}$ have a common left inner (or lossless) divisor (CLLD) if there exists an inner function $U \in H_{m \times m}^{\infty}$ such that $F_{1}=U F_{3}, F_{2}=U F_{4} . V$ will be the greatest common left inner (or lossless) divisor (GCLLD) if $V=U W$ for any other CLLD $U$. Now suppose that the matrix $\left[F_{1}, F_{2}\right]$ has full rank $m$ a.e. Then, the GCLLD exists and has the following geometric interpretation: it is the unique modulo $a$ constant unitary factor from the right inner function defined by the smallest right invariant subspace of $H_{m}^{2}$ which contains both $F_{1} H_{k}^{2}$ and $F_{2} H_{l}^{2}$ (i.e., $\overline{F_{1} H_{l c}^{2} \vee F_{2} H_{l}^{2}}$ ). $F_{1}, F_{2}$ are left coprime if their GCLLD is $I_{m}$. Similarly $F_{1} \in H_{k \times n}^{\infty}$, $F_{2} \in H_{l \times n}^{\infty}$ have a common right inner (or lossless) divisor (CRLD) if $F_{1}=F_{5} U, F_{2}=F_{6} U$ for some inner function $U \in H_{n \times n}^{\infty}$. $V$ will be the greatest common right inner (or lossless) divisor (GCRLD) if $. V=W U$ for any other CRLD $U$. $F_{1}, F_{2}$ are right coprime if their GCRLD is $I_{n}$. For an inner function $U \in H_{n \times n}^{\infty}$ we can define its determinant det $U$ which is a scalar inner function with the property [26]

$$
(\operatorname{det} U) H_{n}^{2} \subset U H_{n}^{2} \text {. }
$$

The structure of $U$ is very much determined from properties of det $U$. We will need also the following result [11], [12]:

Let $A \in H_{m \times k}^{\infty}, B \in H_{m \times l}^{\infty}$ have a GCLLD $U \in H_{m \times m}^{\infty}$. Then there exist sequences of matrices $\left\{M_{i}\right\}$ in $H_{k \times m}^{\infty}$ and $\left\{N_{i}\right\}$ in $H_{l \times m}^{\infty}$ such that

$$
\lim _{i \rightarrow \infty}\left(A M_{i}+B N_{i}\right)=U
$$

the limit standing for either column wise $L^{2}\left(d \omega /\left(1+\omega^{2}\right)\right)$ convergence on the imaginary axis or (weaker) uniform convergence on compact subsets of the ORP [11].

Potapov [32] has studied an even larger class of functions. Let

$$
I=\left[\begin{array}{cc}
1_{p} & 0 \\
0 & -1_{q}
\end{array}\right]
$$

We will say that a matrix function $A(p)$ is $J$-contractive in the ORP if for all $p$ there one has

$$
J-\tilde{A} J A \geqslant 0 \text {. }
$$

Because of the indefinite metric introducted by $J$, we have that $A$ need not be analytic in the ORP. $A$ can have both poles and zeros in the ORP, yet they cannot be arbitrarily distributed. There is a matrix closely related to $A$ which is actually analytic (belongs to $H_{(p+q) \times(p+q)}^{\infty}$ and contractive, namely:

$$
A_{1}=\left(A P-P^{\perp}\right)^{-1}\left(P-A P^{\perp}\right)
$$

where

$$
P=\left[\begin{array}{ll}
1_{p} & 0 \\
0 & 0_{q}
\end{array}\right] \quad P^{\perp}=\left[\begin{array}{ll}
0_{p} & 0 \\
0 & 1_{q}
\end{array}\right]
$$

(supposing the matrix formed by the $p$ first rows and columns in $A$ nonsingular which will always be the case in the sequel). For $J$-contractive functions which are $J$-unitary on the imaginary axis Potapov has obtained factorizations similar to the Blaschke part-singular part factorizations of inner functions.

We terminate this preliminary section with a note on semigroup theory. Let $F(t), t \geqslant 0$ be a family of bounded operators on $\mathcal{F}$ with the properties

$$
\begin{aligned}
F(t) F(s) & =F(t+s), \quad t, s \geqslant 0 \\
\lim _{t \rightarrow 0} F(t) x & =x
\end{aligned}
$$

where the limit is in the norm of $\mathcal{H}$ sense. Such a family us called a $C_{0}$ semigroup of bounded operators on $\mathcal{K}$. If we let

$$
A x=\lim _{h \rightarrow 0} \frac{F(h) x-x}{h}
$$

for those $x$ for which the strong limit exists, we have defined a closed linear operator on $\mathcal{H}$ and its domain $\mathscr{D}(A)$ (which is dense). An $A$ so defined is called the infinitesimal generator of the semigroup and we write $F(t)=e^{A t}$. The Hille-Yosida theory [39] completely characterizes $C_{0}$ semigroups and their generators.

\section{Linear MUltivariable-Distributed Systems}

In this part of the paper, we analyze state-space and frequency-domain models for linear multivariable constantdistributed systems. The distributed systems we have in mind 
include systems governed by certain classes of partial differential equations. The theory may seem at first abstract, but it has two important advantages: i) it allows the parallel analysis of state-space and transfer function models in some detail; ii) it is in all aspects a natural generalization of the well-known state-space and transfer-function theory of linear constant lumped systems. It is i) that permits the development of an elegant and intuitively clear decomposition theory, which reflects many desirable, from the engineering point of view, properties. Both i) and ii) provide, in our opinion, the basis for a systematic development of approximate models which could be utilized in suboptimal design for various practical applications. We restrict our attention to distributed systems that have a state-space representation, with the state space having the structure of a Hilbert space. Such systems arise wery naturally in various problems of continuum mechanics, linear viscoelasticity, linear wave propagation, distributed networks and linear diffusion (heat conduction, etc.) when energy constraints are present. This restriction is justified, on one hand, by the plethora of distributed systems steming from practical applications that are included in this class, and on the other hand, by the rather detailed analysis that one can develop for these systems.

In addition to their potential use in design, (a very good instance of this is described in Section III of this paper), the methods described here can eventually lead to a satisfactory (from the implementation point of view) treatment of linear filtering theory for distributed systems via spectral factorization or innovations and the development of efficient computational algorithms for the design of suboptimal filters and suboptimal controllers. The reason for this, is that the mathematical tools used here are the same with those developed by Wold [40], Wiener [41], Massani [42], Kolmogorov [43][45], and Krein [46], [47] for the now classical filtering theory of stationary time series with nonrational spectra.

\section{II.1. State-Space Models}

The state-space models that we analyze are mainly of the form

$$
\begin{aligned}
\frac{d x(t)}{d t} & =A x(t)+B u(t) \\
y(t) & =C x(t)
\end{aligned}
$$

where $x(t) \in \mathfrak{X}$, a Hilbert space, $A$ is a possibly unbounded operator on $\mathcal{X}$ which generates a strongly continuous semigroup of bounded operators on $X[39], u(t) \in \mathcal{U}$ a finiteimensional Hilbert space (which we will identify with $\boldsymbol{C}^{n}$ ) and $y(t) \in \mathcal{Y}$ a finite-dimensional Hilbert space (which we will identify with $\mathbb{C}^{m}$ ). The derivative is usually considered in the distributional or weak sense [39]. This is equivalent to considering the integral form of (II.1.1),

$$
y(t)=C e^{A\left(t-t_{0}\right)} x\left(t_{0}\right)+\int_{t_{0}}^{t} C e^{A(t-\tau)} B u(\tau) d \tau
$$

whereby $e^{A t}$ denotes the semigroup generated by $A$ (and is a generalization for distributed systems of the wellknown transition matrix of lumped systems). The reader will certainly recognize that in applications $A$ will often take the form of a linear partial differential operator on a domain $\Omega$ of some Euclidean space [48] or the form of an integral operator [56]. We will denote by $\mathcal{D}(A)$ the domain of the unbounded oper- ator $A$ and by $\mathcal{L}\left(\mathfrak{X}_{1}, \mathfrak{X}_{2}\right)$ the space of all continuous linear operators mapping the Hilbert space $\mathscr{X}_{1}$ into the Hilbert space $X_{2}$. In (II.1.1) $B \in \mathcal{L}(\mathcal{U}, \mathcal{X})$ and $C \in \mathcal{L}(\mathcal{X}, \mathcal{Y})$. Typical input functions for this type of systems are square integrable U-valued functions.

By the usual arguments on linearity and time invariance, the input-output description of such systems in the time domain is completely described by the weighting pattern:

$$
T(t)=C e^{A t} B
$$

which is an $m \times n$ matrix valued function. Clearly the zeroinitial condition response is given by

$$
y(t)=\int_{t_{0}}^{t} T(t-\tau) u(\tau) d \tau .
$$

By the use of Laplace transforms the input-output description of such systems in the frequency domain is characterized by the properties of the transfer function:

$$
\hat{T}(p)=C(p I-A)^{-1} B
$$

which is the Laplace transform of $T$. Caution should be exercised in interpreting (II.1.5) here, because it is only valid in certain regions of the complex plane, since it involves nonrational elements (compare with the lumped case). The operator $(p I-A)^{-1}$ is the resolvent operator [39] of the operator $A$.

Considering the triple of operators $(A, B, C)$ we will say that they form a regular realization of the weighting pattern $T$ whenever $A$ generates a semigroup, $B, C$ are bounded and (II.1.3) holds. The system theoretic interpretation of this is well known. $T$ represents input-output relations while $(A, B$, $C$ ) an actual dynamical model. It is also important to consider cases where the operator $C$ may be unbounded. We consider such a class here. The triple $(A, B, C)$ is a balanced realization when (II.1.3) holds but now the range of $B$ is included in the domain of $A$ while $C$ is linear and $A$-bounded [57] (i.e., $\mathfrak{D}(A) \subseteq \mathscr{D}(C)$ and $\|C x\|_{Y} \leqslant k_{1}\|A x\|_{X}+k_{2}\|x\|_{X}$ for some positive constants $k_{1}, k_{2}$ and all $x \in \mathcal{D}(A)$ ). Note that in the case of a balanced realization $B$ is bounded but $C$ is not necessarily.

Representative examples of various types of realizations (systems) that usually arise in practical applications may be found in [2], [48], [49], [53]. The physical distinction between regular and balanced realizations is as follows. When the controls are distributed (i.e., are applied in the whole spatial region) and the observations are also distributed (typically a weighted average) the resulting realization is regular. Whenever the controls are distributed but the observations are restricted on the boundary of the spacial region the resulting realizations are often balanced. We would like to point out that our framework includes hereditary differential systems [54]-[56]. For further discussion of balanced versus regular realizations we refer in particular to [2].

Although balanced and regular realizations can describe quite different physical situations, the classes of input-output relations they characterize coincide, as the following theorem indicates.

Theorem II.1.1: Let $T$ be an $m \times n$ matrix weighting pattern. Then $T$ has a balanced realization if and only if it has a regular realization. Moreover the infinitesimal generators in both realizations can be taken to be the same.

Proof: This is an easy generalization of Baras and Brockett [3, Theorem 3] (see also [8]).

In the effort to choose simplified models one defines as usual a realization $(A, B, C)$ to be reachable if $B^{*} e^{A^{*} t} x=0$ 
for $t \geqslant 0$, (the asterisk here denotes adjoint) implies $x=0$, and observable if $C e^{A t} x=0$ for $t \geqslant 0$ implies $x=0$ (in case of a balanced realization this must be true only for all $x \in \mathcal{D}(A)$ ). A realization is canonical whenever it is reachable and observable. Although this concept is a particularly useful one in the analysis of lumped systems, this is not so for distributed systems, as we shall see later in this part of the paper. Nevertheless given a realization (regular or balanced) it is an easy matter to obtain a canonical one. This is described in the following theorem which we give without proof since it is a straightforward generalization of previous results, Balakrishnan [58], Baras [5].

Theorem II.1.2: Let $(A, B, C)$ be a regular realization of a matrix weighting pattern $T$ with state space $X$. Let be the orthogonal complement in $\mathcal{X}$ of the subspace $\mathbb{N}_{1}=\{x \in \mathcal{X}$; $C e^{A t} x=0$ for $\left.t \geqslant 0\right\}$ and $P_{n}$ the associated orthogonal projection. Let $\pi$ be the orthogonal complement in $r$ of the subspace $r_{1}=\left\{x \in \pi, P_{\pi} B^{*} e^{A^{*} t} x=0\right.$ for $\left.t \geqslant 0\right\}$ and let $P_{\pi}$ be the associated orthogonal projection. Then $\left\{\left.P_{r} A\right|_{r}, P_{r} B\right.$, $\left.C P_{\pi}\right\}$ is a canonical regular realization for $T$, with state space $\pi$.

It can be easily shown that the associated balanced (regular) realization to a regular (balanced) realization is canonical whenever the regular (balanced) realization is [5]. This theorem therefore provides also a reduction for balanced realizations.

The importance of canonical realizations in lumped systems, stems primarily from the so called state-space isomorphism theorem [59] and its various consequences. This result states that any two canonical realizations of the same weighting pattern are similar, that is they differ only by a choice of basis in the state space. To date however no such satisfactory result in the theory of state space models for distributed systems has been discovered. The intuitive reason is that canonical realizations do not precisely capture the intrinsic properties of the distributed system. For a complete and detailed discussion of the current status of this important problem, examples, counterexamples and special cases, we refer the reader to Baras et al. [4]. We give here a fairly general version of the state space isomorphism theorem for the class of systems we consider, which is of importance for the rest of this part of the paper. Consider the reachability and observability Grammians for (II. 1.1)

$$
\begin{aligned}
& W\left(t_{1}\right)=\int_{0}^{t_{1}} e^{A t} B B^{*} e^{A^{*} t} d t \\
& M\left(t_{1}\right)=\int_{0}^{t_{1}} e^{A^{*} t} C^{*} C e^{A t} d t .
\end{aligned}
$$

Following Helton [24], we say that a system $(A, B, C)$ is exactly reachable if the limit as $t_{1}$ goes to infinity of $W\left(t_{1}\right)$ exist as a bounded and boundedly invertible operator and is exactly observable if the limit as $t_{1}$ goes to infinity of $M\left(t_{1}\right)$ exists as a bounded and boundedly invertible operator, (see also in relation Balakrishnan [58, p. 109]). The following theorem ([4, Theorem 5]) is the continuous time version of a result originally due to Helton for discrete time systems [24].

Theorem II.1.3: Let $(A, B, C)$ and $(F, G, H)$ be two reachable (respectively observable) realizations of the same weighting pattern which are also exactly observable (respectively exactly reachable). Then the two realizations are similar, i.e., there exists a bounded and boundedly invertible operator $P$ such that

$$
P A=F P \quad P B=G \quad C=H P .
$$

Notice that the requirement of exact observability has a very intuitive interpretation. It expresses the property that the initial condition (or state) be determined asymptotically in a stable way, from the knowledge of the input and output histories.

\section{II.2. Vectorial Hardy Spaces and Transfer Function Models}

The main question studied in this section, is the characterization of matrix weighting patterns that admit regular realizations.

The first result in this direction is rather elementary, but it provides explicitly the limitations imposed on the weighting pattern when it admits such realizations. For various results of this type see also Baras and Brockett [3], Fuhrmann [17], Baras [2], Helton [24], and Balakrishnan [60]. The theorem that follows is a straightforward generalization of Baras and Brockett [3, Theorem 4] arid we omit the proof.

Theorem II.2.1: Let $T$ be an $m \times n$ matrix weighting pattern. Then if $T$ admits a regular realization it is continuous and of exponential order (i.e., each element of the matrix is like that). A sufficient condition for the existence of a regular realization is that every element of $T$ be locally absolutely continuous and that the derivative of $T$ be of exponential order.

This theorem expresses time domain properties implied by the existence of a regular realization. To obtain a more satisfactory theory we need to explicitly work in the frequency domain.

Theorem II.2.2: Let $T$ be a matrix weighting pattern which is continuous and such that the Laplace transform $\hat{T}$ is analytic. A sufficient condition for $T$ to have a regular realization is that $\hat{T}$ has a factorization $\hat{T}(j \omega)=C(j \omega)^{*} B(j \omega)$ a.e. on the imaginary axis, where $\dot{C} \in H^{2}\left(\mathcal{L}\left(\mathbb{C}^{m}, N\right)\right)\left(\right.$ or $C \in K^{2}\left(\mathcal{L}\left(\mathbb{C}^{m}, N\right)\right.$ ) and $B \in H^{2}\left(\mathcal{L}\left(\mathbb{C}^{n}, N\right)\right)$ (respectively $B \in K^{2}\left(\mathcal{L}\left(\mathbb{C}^{n}, N\right)\right.$ ) where $N$ is an auxiliary Hilbert space.

Proof: We follow Baras [8]. To produce a regular realization, consider as state space $\mathcal{X}$ the space $H^{2}(N)$, as $G$ the operator

$$
G: \mathbb{C}^{n} \longrightarrow X ;(G u)(j \omega)=B(j \omega) u
$$

as $e^{F t}$ the semigroup

$$
e^{F t} x=P_{H^{2}(N)} M_{e}^{j \omega t} x
$$

where $P_{H^{2}}(N)$ is the projection from $L^{2}((-j \infty, j \infty) ; N)$ onto. $H^{2}(N)$, and $M_{e^{j \omega t}}$ is the operator "multiplication by $e^{j \omega t}$. Finally as $H$ consider the operator

$$
H: X \longrightarrow C^{m} ; H x=\frac{1}{2 \pi} \int_{-\infty}^{\infty} C^{*}(j \omega) x(j \omega) d \omega .
$$

Clearly $G \in \mathscr{L}\left(\boldsymbol{C}^{n}, \mathcal{X}\right), H \in \mathcal{L}\left(\mathcal{X}, \mathscr{C}^{m}\right)$, and $e^{F t}$ is a $C_{0}$-semigroup. To complete the proof observe that $\hat{T}$ is actually in $H_{m \times n}^{1}$, so that we have, pointwise:

$$
\begin{aligned}
H e^{F t} G u & =\frac{1}{2 \pi} \int_{-\infty}^{\infty} e^{j \omega t} C^{*}(j \omega) B(j \omega) u d \omega \\
& =\frac{1}{2 \pi} \int_{-\infty}^{\infty} e^{j \omega t} \hat{T}(j \omega) u d \omega=T(t) u, \quad \text { for all } u \in \mathbb{C}^{n} .
\end{aligned}
$$


In the other case, i.e., when $C \in K^{2}\left(\mathcal{L}\left(\mathbb{C}^{m}, N\right)\right)$ and $B \in K^{2}$ $\left(\mathscr{L}\left(C^{n}, N\right)\right)$ the proof is similar and utilizes $K^{2}(N)$ as state space.

We produced a realization whose dynamics are described by the translation semigroup; indeed by use of Fourier transforms we can easily see that $e^{F t}$ is just the semigroup of left translations,

$$
f(\sigma) \stackrel{e^{F t}}{\longrightarrow} f(\sigma+t), \quad \text { for } t \geqslant 0
$$

on $L^{2}(N)$. This translation realization plays a fundamental role in this part of the paper.

The discussion that follows has two aims. On one hand, we want to indicate under what additional assumptions the factorization condition of Theorem II.2.2 becomes also necessary. On the other hand, we would like to investigate relations between this factorization condition and qualitative properties of the system.

We observe first that Theorem II.2.2 is a generalization of Theorem II.2.1. Indeed the sufficient conditions of Theorem II.2.1 imply that after multiplication by a proper exponential factor, $T$ and $(d / d t) T$ are in $L^{2}\left(\mathcal{L}\left(C^{n}, C^{m}\right)\right)$. Therefore if in addition $T(0)=0$ we have the factorization

$$
\hat{T}(j \omega)=\left((1+j \omega)^{-1} I_{m}\right)^{*}(1-j \omega) \hat{T}(j \omega)
$$

where the auxiliary Hilbert space is $N=\mathbb{C}^{m}$. In case $T(0) \neq 0$, the problem can be reduced to the previous one, and solved using the above factorization for an auxiliary weighting pattern.

Let us suppose now, that we know a priori that $T$ comes from a regular realization which is dissipative, in the sense that the operator $A$ is dissipative, i.e.,

$$
\langle A x, x\rangle+\langle x, A x\rangle \leqslant 0, \quad \text { for } x \in \mathcal{D}(A)
$$

and globally asymptotically stable, in the sense that

$$
\lim _{t \rightarrow \infty}\left\|e^{A t} x\right\|_{X}=0
$$

for all $x \in \mathcal{X}$, (the norm and inner product here are those of $X)$. In this case following [52], we consider the new norm $\|x\|_{N}^{2}=-\langle A x, x\rangle-\langle x, A x\rangle$ for $x \in D(A)$. Then for $x \in D(A)$, $e^{A t} x \in \mathcal{D}(A)$ for $t \geqslant 0$ and

$$
\left\|e^{A t} x\right\|_{N}^{2}=-2 \operatorname{Re}\left\langle e^{A t} x, A e^{A t} x\right\rangle=-\frac{d}{d t}\left\|e^{A t} x\right\|_{x}^{2} .
$$

Therefore,

$$
\int_{0}^{\infty}\left\|e^{A t} x\right\|_{N}^{2}=\|x\|_{x}^{2}
$$

That is, if we let $N$ denote the completion of $D(A)$ under the new norm, then the map

$$
P: X \longrightarrow L^{2}(N) ; x \longmapsto h(\sigma)=e^{A \sigma} x
$$

is an isometry. Moreover,

$$
P e^{A t} x=e^{F t} P x
$$

where $e^{F t}$ is the left translation semigroup on $L^{2}(N)$. Since $P$ is an isometry its range $R(P)$ is closed, in fact it is a left translation invariant subspace of $L^{2}(N)$, which we denote by $\mathscr{X}_{1}$. So $P$ as a map from $X_{\text {to }} \mathscr{X}_{1}=R(P)$ has a bounded inverse. Therefore, we obtain a regular realization with state space $\mathfrak{X}_{1}$,
$e^{F t}=$ left translation semigroup, $G=P B$ and $H=C P^{-1}$. Apply ing Fourier transforms $\mathcal{F}$, we get a regular realization with state space $\mathfrak{X}_{2}=\mathcal{F}^{\mathscr{X}_{1}}$ (a subspace of $H^{2}(N)$ ):

$$
\begin{aligned}
e^{F_{1} t} x_{2} & =P_{H^{2}(N)} M_{e^{j \omega t}} x_{2} \\
G_{1} & =\mathcal{F} P B: \mathbb{C}^{n} \longrightarrow H^{2}(N) \\
H_{1} & =C P^{-1} \mathcal{F}^{-1}: H^{2}(N) \longrightarrow \mathbb{C}^{m}
\end{aligned}
$$

Then there exists $B_{1} \in H^{2}\left(\mathcal{L}\left(\mathscr{C}^{n}, N\right)\right)$ such that

$$
\left(G_{1} u\right)(j \omega)=B_{1}(j \omega) u \quad \text { for all } u \in \mathbb{C}^{n}
$$

and $C_{1} \in H^{2}\left(\mathcal{L}\left(\mathbb{C}^{m}, N\right)\right)$ such that

$$
H_{1} x=\frac{1}{2 \pi} \int_{-\infty}^{\infty} C_{1}^{*}(j \omega) x(j \omega) d \omega \quad \text { for all } x \in H^{2}(N) .
$$

Therefore,

$$
\hat{T}(j \omega)=C_{1}^{*}(j \omega) B_{1}(j \omega) \text { a.e. }
$$

which is clearly a factorization of the type appearing in Theorem II.2.2.

In another direction, suppose that a matrix weighting pattern is square integrable and has a reachable and exactly observable regular realization (see Section II.1).

The operator

$$
\begin{aligned}
H_{T}: L_{n}^{2}(0, \infty) & \longrightarrow L_{m}^{2}(0, \infty) \\
\left(H_{T} u\right)(t) & =\int_{0}^{\infty} T(t+\sigma) u(\sigma) d \sigma
\end{aligned}
$$

which is well defined and bounded under our assumption, is the Hankel operator associated to $T$, and is the generalization of the standard Hankel matrix of lumped systems [59]. Consider the range $R\left(H_{T}\right)$ of the Hankel operator. Clearly it is invariant under left translations. Then a realization of $T$ is given by

$$
\text { state space } \begin{aligned}
\mathcal{X} & =\text { closure of } R\left(H_{T}\right)=\overline{R\left(H_{T}\right)} \\
e^{F t} & =\text { left translation semigroup on } X \\
(G u)(\sigma) & =T(\sigma) u \\
H x & =x(0) .
\end{aligned}
$$

It is easy to show that our assumptions imply that $H \in \mathcal{L}\left(\mathcal{X}, C^{m}\right),[58$, p. 109]. Therefore, the above realization is clearly a regular realization. Now $G^{*} e^{F^{*} t} x=0, t \geqslant 0$, implies

$$
\int_{t}^{\infty} \tilde{T}(\sigma) x(\sigma-t)=0, \quad \text { for all } t \geqslant 0
$$

( $\tilde{T}$ is the Hermitian conjugate of $T$ ). Therefore,

$$
\int_{0}^{\infty} \tilde{T}(t+\tau) x(\tau) d \tau=0, \quad \text { for all } t \geqslant 0
$$

which implies $x \perp \overline{R\left(H_{T}\right)}$ and thus $x=0$. That is this realization is reachable.

Similarly it is easy to see that

$$
\int_{0}^{\infty} e^{F^{*} t} H^{*} H e^{F t} d t x=x
$$

and, therefore, this realization is exactly observable. More- 
over by Theorem II.1.3 all reachable and exactly observable realizations of $T$ differ from the one described above by a similarity transformation. Applying Fourier transforms in (II.2.1) we have the Hankel operator associated to $\hat{T}$

$$
\begin{aligned}
H_{\hat{T}}: H_{n}^{2} & \longrightarrow H_{m}^{2} \\
H_{\hat{T}} \hat{u} & =P_{H_{m}^{2}} M_{\hat{T}} \sigma_{-} \hat{u}
\end{aligned}
$$

where $\left[M_{\hat{T}} \hat{u}\right](j \omega)=\hat{T}(j \omega) \hat{u}(j \omega)$ (i.e., multiplication by $\hat{T}$ ). We also obtain the following realization in $H_{m}^{2}$.

$$
\begin{aligned}
\text { state space } X & =\overline{R\left(H_{\hat{T}}\right)} \\
e^{A t} x & =P_{X} M_{e}^{j \omega t} x \\
(B u)(j \omega) & =\hat{T}(j \omega) u \\
C x & =\frac{1}{2 \pi} \int_{-\infty}^{\infty} x(j \omega) d \omega .
\end{aligned}
$$

Then since $B \in \mathcal{L}\left(\mathbb{C}^{n}, X\right)$ and $C \in \mathcal{L}\left(\mathscr{X}, \mathscr{C}^{m}\right)$ and $\mathcal{X} \subset H_{m}^{2}$ it is straightforward to show (exactly as in the previous dissipative case) that we have a factorization $\hat{T}(j \omega)=F_{1}(j \omega)^{*} F_{2}(j \omega)$ a.e. as described in Theorem II.2.2. The auxiliary Hilbert space is finite dimensional. Summarizing we have the following.

Theorem $I I .2 .3$ : Let $\hat{T}$ be a transfer function matrix. If either

a) $\hat{T}$ has a dissipative and stable realization, or

b) $\hat{T}$ is in $H_{m}^{2} \times_{n}$ and has a reachable and exactly observable realization

then $\hat{T}(j \omega)=C^{*}(j \omega) B(j \omega)$ a.e. where $B \in H^{2}\left(\mathcal{L}\left(\mathbb{C}^{n}, N\right)\right)$ and $C \in H^{2}\left(\mathcal{L}\left(\mathbb{C}^{m}, N\right)\right)$ and $N$ is an auxiliary Hilbert space.

The realization described in (II.2.2) or (II.2.4) will be called the restricted translation realization (or restricted shift realization). Let us observe that one can formally consider this realization for any $\hat{T} \in H_{m \times n}^{\infty}$. Then however, the realization is neither regular nor balanced. We note also that the factorization of Theorems II.2.2. and II.2.3. is akin but a little different than the coprime factorization of Section II.4, in that it requires the factors to have elements in $H^{2}$. This is intrinsic to the continuous time case, while in the discrete time case none of the latter difficulties arise since ${ }_{d} H^{\infty} \subset{ }_{d} H^{2}$.

\section{II.3. Invariant Subspaces and Modules}

We saw at several instances in the previous sections how invariant subspaces appear naturally in the analysis of statespace or transfer function models. The purpose of this section is to make this more explicit, and then to utilize the natural tools of invariant subspace theory to develop a number of important results. If the weighting pattern $T$ is an element of $L_{m \times n}^{2}(0, \infty) \cap L_{m \times n}^{1}(0, \infty)$, it is quite clear that the inputoutput map $f_{\Sigma}$ of the system (II.1.1) is a linear continuous map

$$
f_{\Sigma}: L_{n}^{2}(-\infty, \infty) \longrightarrow L_{m}^{2}(-\infty, \infty)
$$

which commutes with translations

$$
f_{\Sigma} \sigma_{\tau}=\sigma_{\tau} f_{\Sigma}
$$

and is causal, i.e., $f_{\Sigma}\left(L_{n}^{2}(0, \infty)\right) \subseteq L_{m}^{2}(0, \infty)$.

Conversely suppose that we are given a linear continuous map $f_{\Sigma}$ between $L_{n}^{2}(-\infty, \infty)$ and $L_{m}^{2}(-\infty, \infty)$. In order for $f_{\Sigma}$ to be the input-output map of a causal, time constant system, $f_{\Sigma}$ must commute with translations and $f_{\Sigma}\left(L_{n}^{2}(0, \infty)\right) \subseteq$ $L_{m}^{2}(0, \infty)$. Then in the Fourier domain, the input-output map
$\hat{f}_{\Sigma} \triangleq F_{f_{\Sigma}} \mathcal{F}^{-1}$ is described by (Bochner-Chandrasekharan theorem [38]):

$$
\begin{gathered}
\hat{f}_{\Sigma}: L_{n}^{2}(-j \omega, j \omega) \longrightarrow L_{m}^{2}(-j \omega, j \omega) \\
\hat{f}_{\Sigma} \hat{u}=\hat{T} \hat{u}=\hat{T} \mathfrak{F} \hat{u}
\end{gathered}
$$

where $\hat{T} \in H_{m \times n}^{\infty}$.

A useful and equivalent description is given by the restricted map.

$$
f: \Omega=L_{n}^{2}(-\infty, 0] \longrightarrow L_{m}^{2}[0, \infty)=\Gamma .
$$

Kalman used $f$ [62] in his development of the module theory of linear, constant finite-dimensional discrete-time systems. This theory can be extended to the present setting. Following Kalman [62], recall that the map $f$ is defined as follows: for $\omega \in \Omega$

$$
\begin{aligned}
f(\omega)(t) & =f_{\Sigma}(\omega)(t), & & t \geqslant 0 \\
& =0, & & t<0 .
\end{aligned}
$$

Let $\sigma_{t}^{r}$ be the operation of left translation by $t$, followed by restriction on $[0, \infty)$. Then

$$
\sigma_{t}^{r} f=f \sigma_{t}
$$

Clearly in the frequency domain $\hat{f}=\mathcal{F}^{f^{-1}}$ is described by

$$
\begin{aligned}
\hat{f}: K_{n}^{2} & =\hat{\Omega} \longrightarrow \hat{\Gamma}=H_{m}^{2} \\
\hat{f} & =P_{H_{m}^{2}} M_{\hat{T}} .
\end{aligned}
$$

Therefore, we have the following commutative diagram (II.3.3)

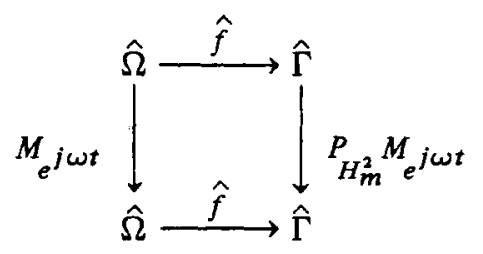

where as usual $M_{e^{j \omega t}}$ is multiplication by $e^{j \omega t}$. Clearly $\hat{\Omega}$ admits a module structure with the ring being $K^{\infty}$. The scalar multiplication is

$$
h \cdot \hat{u}=\left[\begin{array}{c}
h \hat{u}_{1} \\
h \hat{u}_{2} \\
\vdots \\
h \hat{u}_{n}
\end{array}\right], \quad h \in K^{\infty}, \hat{u} \in \hat{\Omega} .
$$

Similarly $\hat{\Gamma}$ admits a $K^{\infty}$-module structure where the scalar multiplication is defined by

$$
h \square \hat{\gamma}=P_{H_{m}^{2}}\left[\begin{array}{c}
h \hat{\gamma}_{1} \\
h \hat{\gamma}_{2} \\
\vdots \\
h \hat{\gamma}_{n}
\end{array}\right] .
$$

Let

$$
\chi(j \omega)=\frac{1+j \omega}{j \omega-1}
$$


clearly $\chi \in K^{\infty}$. Then it follows from Nagy-Foias [31 p. 142] (relations between semigroups and cogenerators) that the above diagram commutes if and only if the following diagram commutes:

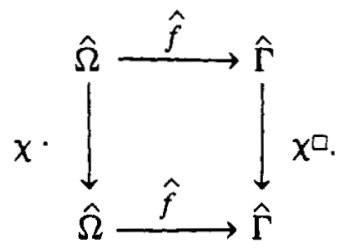

Since $K^{\infty}$ is the bounded pointwise a.e. closure of polynomials in $\chi[29$, p. 107] we conclude that (see also [14]):

Theorem II.3.1: The restricted input-output map of a linear multivariable distributed system in this class (i.e., (II.3.1)) $\hat{f}$, is a $K^{\infty}$-homomorphism (in the frequency domain and with scalar multiplications defined as in (II.3.6), (II.3.7)).

It follows from diagram (II.3.5) that the set of Nerode equivalence classes of $f$ is isomorphic with the quotient module $\hat{\Omega} / \operatorname{Ker} \hat{f}$. Thus the following corollary.

Corollary II.3.2: The "natural" state set $\hat{\Omega} / \operatorname{Ker} \hat{f}$ of a linear multivariable distributed system in this class, admits the structure of a $K^{\infty}$-module.

Other module structures on input and output spaces have been defined by Kamen [65] and Kalman-Hautus [85] in a way appropriate to the topologies considered by these authors. The detailed results available about the structure of the Banach algebras $H^{\infty}$ and $H_{k \times k}^{\infty}$ (and therefore $K^{\infty}$ and $K_{k \times k}^{\infty}$ ) [29] , [30] make possible a detailed structural analysis for this class of distributed systems [64].

It is of ten helpful to consider together with the given system $\Sigma$, defined by the map $f_{\Sigma}$ the dual system $\Sigma *$ which has input-output map

$$
\begin{gathered}
f_{\Sigma^{*}}=\sigma_{-} f_{\Sigma}^{*} \sigma_{-} \\
f_{\Sigma} \cdot: L_{m}^{2}(-\infty, \infty) \longrightarrow L_{n}^{2}(-\infty, \infty)
\end{gathered}
$$

where $f_{\Sigma}^{*}$ is the Hilbert space adjoint of $f_{\Sigma}$. It is easy to show that $\Sigma^{*}$ is a linear time constant system with transfer function $\widetilde{\hat{T}}(-j \omega)=\sigma_{-} \widetilde{\hat{T}}(j \omega)$. It is trivial to see that if $(A, B, C)$ is a regular realization for $f_{\Sigma}$ then $\left(A^{*}, C^{*}, B^{*}\right)$ is a regular realization for $f_{\Sigma *}$. However, no such complete duality exists for the other types of realizations we discussed in Section II.1. Moreover if $\hat{T} \in H_{m \times n}^{2} \cap H_{m \times n}^{\infty}$ and $\hat{T}(j \omega)=C^{*}(j \omega) B(j \omega)$ we have immediately that $\sigma_{-} \hat{T} \in H_{n \times m}^{2} \cap H_{n \times m}^{\infty}$ and

$$
\sigma_{\_} \tilde{\hat{T}}(j \omega)=B^{*}(-j \omega) C(-j \omega)=\left[\left(\sigma_{-} B\right)^{*} \sigma_{-} C\right](j \omega)
$$

and clearly $\sigma_{-} B \in K^{2}\left(\mathcal{L}\left(\mathbb{C}^{n}, N\right)\right)$ while $\sigma_{-} C \in K^{2}\left(\mathcal{L}\left(\mathbb{C}^{m}, N\right)\right)$ That is duality is built in the factorization condition of Theorem II.2.2. Finally let us denote by $f_{*}$ the restricted map of $f_{\Sigma}$ * (see (II.3.2)).

Using the Hankel operator $H_{\hat{T}}$ associated with $\hat{f}$ (or $\hat{T}$ ) (II.2.3), we describe now two natural realizations of $\hat{f}$ (or T) (see [62] for the corresponding constructions in the lumped case). We have from (II.3.5) the following commutative diagram for $H_{\hat{T}}$ :

$$
M_{e}-\left.j \omega t\right|_{H_{n}^{2} \stackrel{H_{\hat{T}}}{\longrightarrow} H_{m}^{2}} ^{H_{n}^{2}}{ }_{H_{m}^{2}}^{H_{\hat{T}}} M_{e}^{j \omega t}
$$

From the diagram it is clear that $\hat{\pi}=\operatorname{Ker}\left(H_{\hat{T}}\right)$ is a right invariant subspace of $H_{n}^{2}$ and $\hat{\pi}=$ Range $\left(H_{\hat{T}}\right)^{\perp}$ is a right invariant subspace of $H_{m}^{2}$. So to every input-output map in this class (II.3.1) (or transfer function) we have naturally associated two right invariant subspaces. The orthogonal complements of these subspaces are left invariant subspaces and are the state spaces of the realizations we proceed now to describe. The first is given in (II.2.4) and has as state space $\overline{R\left(H_{\hat{T}}\right)}=$ $\hat{r}^{\perp} \subset H_{m}^{2}$. Recall that this is regular whenever $\hat{T}$ is in $H_{m \times n}^{2}$ and has an exactly observable and reachable realization, in which case (II.2.4) is similar to any other such realization. In that case the natural way to construct the second realization is via duality. Towards this end observe that $H_{\tilde{T}}^{*}=H_{\sigma_{-}} \hat{\hat{T}}$. Therefore, we construct (II.2.4) for $\sigma_{-} \tilde{\hat{T}}$ and then take adjoints:

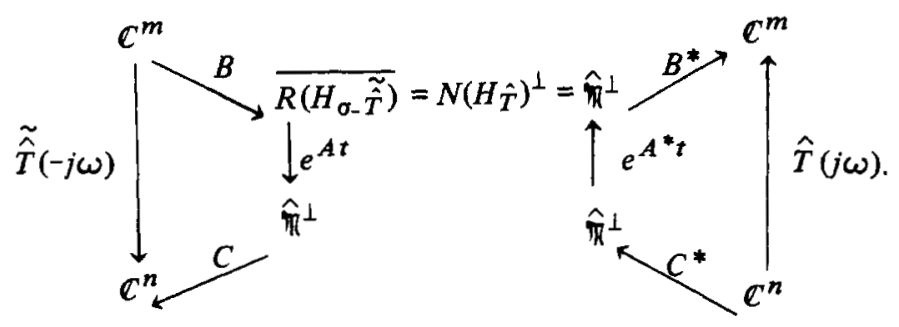

In the above diagram $e^{A t}, B, C$ are as defined in (II.2.4) but for $\sigma_{-} \tilde{\hat{T}}$ now. This second realization described by (II.3.11) has clearly dual properties to those of (II.2.4). So it is regular whenever $\hat{T} \in H_{m \times n}^{2}$ has an exactly reachable and observable realization. Moreover it is itself exactly reachable and observable and by the state-space isomorphism theorem (Theorem II.1.3) any other such realization of $\hat{T}$ is similar to (II.3.11). In the context of shift realizations for discrete time systems, Fuhrmann [18] has obtained specific conditions for a realization to be reachable, exactly reachable, observable or exactly observable. These conditions apply here as well, and we refer the reader to [18] for details.

From the Beurling-Helson-Lax theorem, there exist two functions $Q_{r} \dot{\epsilon} H_{m \times k}^{\infty}, Q_{l}^{\prime} \in H_{n \times c^{\prime}}^{\infty}$, such that $Q_{r}(j \omega), Q_{l}^{\prime}(j \omega)$ are isometries a.e. on the imaginary axis, which describe the right invariant subspaces $\hat{\pi}$ and $\hat{h}$ as

$$
\begin{aligned}
& \hat{\pi}=Q_{r} H_{k}^{2} \\
& \hat{k}=Q_{l}^{\prime} H_{k^{\prime}}^{2} .
\end{aligned}
$$

We note that in general $k \leqslant m, k^{\prime} \leqslant n$. So to every transfer function $\hat{T}$ in this class we associate quite naturally two isometric analytic functions $Q_{r}$ and $Q_{l}^{\prime}$. The importance of these two functions will be demonstrated in the next section. Note that these fünctions are uniquely associated to $\hat{T}$, modulo constant unitary factors.

\section{II.4. Generalized Singularities of Transfer Functions and Spectral Analysis}

In this section, we single out a class of linear multivariable distributed systems for which a fairly detailed frequency domain and state-space theory can be developed. In the analysis of systems like (II.1.1) any available information about the spectral properties of A (i.e., eigenvalues etc.) considerably facilitates the analysis. A very popular method when $A$ is self-adjoint with pure-point spectrum (i.e., only eigenvalues) is via modal analysis (that is eigenfunction expansions). 
Modal analysis (when applicable) not only facilitates the design but clarifies completely the internal structure of the system and provides extremely useful and meaningful approximations, via truncation. Quite often control laws based on approximate knowledge of the natural modes, behave satisfactorily [66]. From the input-output point of view the pertinent information about the natural modes of the system is included in the analytic behavior of $\hat{T}$. So when modal analysis is not applicable, it is clear that state-space models which have operators $A$ with spectral properties faithfully representing the singularities of $\hat{T}$ are quite desirable and useful. Such studies were initiated by Baras and Brockett [3]. See also Fuhrmann [23] and Baras [5], [6], [8]. One of the important properties for the class of systems we mentioned in the beginning of this section is that these systems do have such models, and moreover this property is generic for models that are "minimal" in a certain sense.

In applications the transfer function $\hat{T}$ will be certainly analytic in the ORP but it will have also analytic extensions to portions of the OLP. Only pathological examples will lead to $\hat{T}$ which have the imaginary axis as a natural boundary. We will let $\sigma(\hat{T})$ denote the set of nonanalyticity of $\hat{T}$. Since we are considering nonrational transfer functions, $\sigma(\hat{T})$ will contain not only poles but also essential singularities, branch cuts. We denote by $\sigma(A)$ the spectrum of the operator $\mathrm{A}$ in the realization $(A, B, C)$ of $\hat{T}$. It is a well-known fact with many important consequences in the lumped systems theory, that in every canonical realization of $\hat{T}, \sigma(\hat{T})$, i.e., the poles of $\hat{T}$, coincide with $\sigma(A)$, i.e., the eigenvalues of $A$, multiplicities counted [59]. That this is not so in distributed systems has been demonstrated by counterexamples in Baras and Brockett [3], Baras [2] , Fuhrmann [17]. Here is another one from [6]: Consider the equation describing the temperature of a long bar, along the surface of which heat transfer takes place to the environment

$$
\frac{\partial x(t, z)}{\partial t}=\frac{\partial^{2} x(t, z)}{\partial z^{2}}-x(t, z) .
$$

The initial condition is $x(0, z)=0$ and the boundary conditions are $x(t, 0)=u(t)$ (the control), $\lim _{z \rightarrow \infty} x(t, z)=0$. The output is the temperature at the point $z=1$. The weighting pattern is

$$
T(t)=e^{-t} \frac{1}{2 \sqrt{\pi t^{3}}} e^{-1 / 4 t}
$$

and the transfer function $\hat{T}(p)=e^{-\sqrt{p+1}}$ where we choose the branch which gives $\operatorname{Re}(\sqrt{p})>0$ whenever $\operatorname{Re} p>0$, and the branch cut is along the negative real axis. Then the following realization:

$$
\begin{aligned}
X & =L_{2}[0, \infty) \\
e^{A t} & =\text { left translation semigroup restricted on }[0, \infty) \\
b & =T-\frac{d}{d t} T \\
c x & =\int_{0}^{\infty} g(t) x(t) d t, \quad \text { where } g(t)=e^{-t}
\end{aligned}
$$

is canonical. However $\sigma(A)=$ closed LP while $\sigma(\hat{T})=$ $\{\lambda \in R ;-\infty \leqslant \lambda \leqslant-1\}=\{$ branch cut from -1 to $-\infty\}$. Fol- lowing Baras and Brockett [3] we will say that a realization is spectrally minimal if $\sigma(A)=\sigma(\hat{T})$, for some analytic continuation of $\hat{T}$ (since $\hat{T}$ may have more than one analytic continuation). Here for simplicity we assume that the resolvent set $\rho(A)$ is connected.

The reader should realize by now that whatever properties one can discover for the restricted translation realizations (II.2.4)-(II.3.11), will hold for a quite general class of distributed systems with reasonable properties.

The class of systems we have in mind are those with transfer functions $\hat{T}$ which give rise to functions $Q_{r}, Q_{l}^{\prime}$, which are square matrices, i.e., $k=m, k^{\prime}=n$ in (II.3.12). That is systems for which the right invariant subspaces $\hat{\pi}$ and $\hat{R}$ are of full range. In this case both $Q_{r}$ and $Q_{l}^{\prime}$, are inner functions. The transfer functions $\hat{T}$ that have this property have also several other important properties which we proceed to describe, Such functions have been studied by Baras [3]-[8], Dewilde [10], Douglas et al. [67], Douglas and Helton [25], Fuhrmann [21]. An $m \times n$ matrix valued function $F$ is meromorphic of bounded type in OLP [25] if it has the form $F=G / g$ where $G \in K_{m \times n}^{\infty}$ and $g \in K^{\infty}$. We can always take $g$ to be inner, i.e., $|g(j \omega)|=1$. A function $H$ in $H_{m \times n}^{\infty}$ has a meromorphic pseudocontinuation of bounded type in OLP [25], if there exists an $F$ which is meromorphic of bounded type in OLP and such that

$$
\lim _{\operatorname{Re} \rightarrow 0^{-}} F(p) u=\lim _{\operatorname{Re} p \rightarrow 0^{+}} H(p) u, \quad \text { for all } u \in C^{n} .
$$

This notion is a quite useful generalization of the classical analytic continuation concept for functions $H$ that cannot be continued analytically in OLP. Moreover the singularities of the pseudo-continuation provide a useful substitute for the singularities of $H$ in such a case. Now we have a transfer function $\hat{T}$ such that

$$
\begin{aligned}
& \overline{R\left(H_{\hat{T}}\right)}=\left(Q_{r} H_{m}^{2}\right)^{\perp} \\
& \overline{N\left(H_{\hat{T}}\right)}=Q_{l}^{\prime} H_{n}^{2}
\end{aligned}
$$

$Q_{r}$ and $Q_{l}^{\prime}$ are uniquely determined modulo a constant unitary factor from the right.

From (II.4.1) it follows that

$$
\begin{gathered}
P_{H_{m}^{2}} M_{\hat{T}} K_{n}^{2} \subset\left(Q_{r} H_{m}^{2}\right)^{\perp} \\
\text { So } \tilde{Q}_{r} P_{H_{m}^{2}} M_{\hat{T}} K_{n}^{2} \subset K_{m}^{2} \text { and } \\
\tilde{Q}_{r} M_{\hat{T}} K_{n}^{2}=\tilde{Q}_{r} P_{H_{m}^{2}} M_{\hat{T}} K_{m}^{2}+\tilde{Q}_{r} P_{K_{m}^{2}} M_{\hat{T}} K_{n}^{2} \subset K_{m}^{2} .
\end{gathered}
$$

Therefore, $\widetilde{Q}_{r}(j \omega) \hat{T}(j \omega)=\tilde{P}_{r}(j \omega)$ for some $P_{r} \in H_{m \times n}^{\infty}$. Thus

$$
\hat{T}(j \omega)=Q_{r}(j \omega) \tilde{P}_{r}(j \omega) .
$$

Moreover, it is easy to show that if we require $Q_{r}, P_{r}$ to be right coprime, these factors are unique modulo constant unitary factors. Now $\widetilde{P}_{r} \in K_{m \times n}^{\infty}$ and let $q_{r}=\operatorname{det} Q_{r}$. Then there exists $U_{r} \in H_{m \times m}^{\infty}$ such that

$$
Q_{r} U_{r}=q_{r} I_{m}, \quad \tilde{U}_{r} \in K_{m \times m}^{\infty} \text {, and } \tilde{q}_{r} \in K^{\infty}
$$

and so $Q_{r}$ has the meromorphic pseudo-continuation of bounded type (MPBT) $\tilde{U}_{r} / \tilde{q}_{r}$, and, therefore, $\hat{T}$ has the MPBT $\widetilde{U}_{r} \widetilde{P}_{r} / \widetilde{q}_{r}$. The remarkable fact is that conversely if $\hat{T}$ has a MPBT, then the $Q_{r}$ associated with $R\left(H_{\hat{T}}\right)^{\perp}$ is inner, see [8], $[10],[21]$. Now if $\hat{T}$ has a MPBT, the transfer function of 
the dual system clearly has one. Indeed if $G / g$ is the MPBT for $\hat{T}$, let $G_{1}(p)=\widetilde{G}(-p), g_{1}(p)=\tilde{g}(-p)$, and then clearly $G_{1} / g_{1}$ is a MPBT for $\sigma_{-} \widetilde{\hat{T}}$ (the transfer function of the dual system). But then by duality

$$
\tilde{\hat{T}}(-j \omega)=Q_{l}^{\prime}(j \omega) \tilde{P}_{l}(j \omega)
$$

and so

$$
\hat{T}(j \omega)=P_{l}^{\prime}(-j \omega) \tilde{Q}_{l}^{\prime}(-j \omega)
$$

and by defining $\tilde{P}_{l}(j \omega)=P_{l}^{\prime}(-j \omega)$ and $Q_{l}(j \omega)=\tilde{Q}_{l}^{\prime}(-j \omega)$ we have the factorization

$$
T(j \omega)=\tilde{P}_{l}(j \omega) Q_{l}(j \omega)
$$

where the factors are unique up to a unitary factor, if we require $P_{l}$ and $Q_{l}$ to be left coprime. We summarize (see also $[8],[10],[21])$ as follows.

Theorem II.4.1: The following are equivalent:

i) $\hat{T}$ is such that $R\left(H_{\hat{T}}\right)^{\perp}=Q_{r} H_{m}^{2}$ with $Q_{r}$ inner.

ii) $\hat{T}$ is such that $N\left(H_{\hat{T}}\right)=Q_{l}^{\prime} H_{n}^{2}$ with $Q_{l}^{\prime}$ inner.

iii) $\hat{T}$ has a meromorphic pseudo-continuation of bounded type in OLP

iv) $\underset{\sim}{T}$ admits a left coprime factorization $\hat{T}(j \omega)=$ $\tilde{P}_{l}(j \omega) Q_{l}(j \omega)$ where $Q_{l} \in H_{n \times n}^{\infty}$ and is inner and $P_{l} \in H_{n \times m}^{\infty}$.

v) $\hat{T}$ admits a left coprime factorization $\hat{T}(j \omega)=U_{l}^{1}$ (jw) $\Delta_{l}(-j \omega)$ where $\Delta_{l} \in H_{m \times n}^{\infty}, U_{l}$ inner in $H_{m \times m}^{\infty}$.

vi) $\stackrel{T}{T}$ admits a right coprime factorization $\hat{T}(j \omega)=$ $Q_{r}(j \omega) \widetilde{P}_{r}(j \omega)$ where $P_{r} \in H_{n \times m}^{\infty}$ and $Q_{r} \in H_{m \times m}^{\infty}$ and is inner.

vii) $\hat{T}$ admits a right coprime factorization $T(j \omega)=$ $\Delta_{r}(-j \omega) U_{r}^{-1}(-j \omega)$ where $\Delta_{r} \in H_{m \times n}^{\infty}$ and $U_{r} \in H_{n \times n}^{\infty}$ and inner.

Note also that modulo constant unitary factors these factorizations are related via

$$
\begin{array}{ll}
P_{l}(j \omega)=\tilde{\Delta}_{r}(-j \omega), & Q_{l}(j \omega)=U_{r}^{-1}(-j \omega) \\
Q_{r}(j \omega)=U_{l}^{-1}(-j \omega), & P_{r}(j \omega)=\tilde{\Delta}_{l}(-j \omega) .
\end{array}
$$

So in particular $N\left(H_{\hat{T}}\right)=U_{r} H_{n}^{2}$ in (II.4.1), for such transfer functions.

Such functions are in the center of the new theory and have been given various names in the system theoretic and network literature. Thus Baras [3], [8] and Fuhrmann [21], [22] called them strictly noncyclic motivated by the work of Douglas et al. [67] for the scalar case, while Dewilde [10][12] called them roomy motivated by the "full-range" property of the two important invariant subspaces. We will use in wis paper either terminology, and keep in mind that they represent the same properties. Moreover we would like to emphasize that all properties in Theorem II.4.1 are at the input-output level.

It is clear from the above that two natural state spaces for the restricted translation realizations are determined from the inner functions $Q_{r}$ and $U_{r}$. The structure of these inner functions on one hand provides the spectral analysis of the corresponding semigroups and on the other hand determines the singularities of the transfer function $\hat{T}$. It is this property that will allow us to demonstrate that the restricted translation realizations (II.2.4)-(II.3.11) are spectrally minimal if $\hat{T}$ is strictly noncyclic. By that we mean that the singularities in the closed LP of the MPBT of $\hat{T}$ coincide with the spectrum of the infinitesimal generator $A$ in these realizations. Thus we have an elegant generalization, for this class, of the lumped system theory result. Moreover we shall see in Section III of this paper how these considerations lead to a satisfactory degree theory. To proceed we need the following well-known result, Moeller [68], Lax [52]. The spectrum of the infinitesimal generator of the semi-group 'multiplication by $e^{j \omega t}$, restricted to the left invariant subspace $\left(Q_{r} H_{m}^{2}\right)^{\perp}$ consists of:

a) the points $\mu$ in OLP where $\widetilde{Q}_{r}(-\tilde{\mu})$ has nonnull kernel.

b) the points on the imaginary axis through which $Q_{r}$ cannot be continued analytically to OLP.

Now the meromorphic pseudo-continuation of $\hat{T}$ is $\tilde{Q}_{r} \widetilde{P}_{r} / \tilde{q}_{r}$ where $q_{r}=\operatorname{det} Q_{r}$. So the poles of the MPBT coincide with the zeros of $\tilde{q}_{r}$ in OLP which in turn coincide with the points $\left\{\mu \in \mathrm{OLP}: \tilde{q}_{r}(-\tilde{\mu})=0\right\}=\left\{\mu \in \mathrm{OLP}: \tilde{Q}_{r}(-\tilde{\mu})\right.$ has nonnull kernel $\}$ and with multiplicities counted. By straightforward generalizations from $[8],[52$, p. 72$]$ we deduce that $\hat{T}$ has an analytic continuation through a point in the imaginary axis if and only if $Q_{r}$ does. So we have:

Theorem II.4.2: For any strictly noncyclic transfer function $\hat{T}$ the restricted translation realizations (II.2.4)-(II.3.11) are spectrally minimal.

Note that in practice, where the continuation of $\hat{T}$ will be a classical continuation, these translation models capture the concept of "natural modes" quite satisfactorily. More generally for transfer functions that satisfy the factorization conditions of Theorem II.2.2 one can obtain a spectrally minimal realization, by applying the reduction procedure of Theorem II.1.2 to the translation realization produced in Theorem II.2.2. The necessary arguments are straightforward generalizations of the arguments used in Baras [5], [6] for the scalar case.

Much more work is needed in order to utilize the full strength of invariant subspace methods in the structural analysis of linear multivariable systems. We believe that this approach, being closely related to the harmonic analysis of operators in Hilbert spaces has all the necessary ingredients to provide a complete generalization of the fine structure theory of lumped systems. The reader should compare the connections between Jordan normal forms and rational matrix properties (McMillan-Smith canonical form) and structure of lumped systems [1], [59], [62] with the connections between Jordan models of operators and properties of $H_{m \times m}^{\infty}$ functions which underline the theory presented here. For some more complex problems, e.g., series parallel connections of infinite dimensional systems, frequency domain conditions for controllability and observability for discrete time systems we refer to Fuhrmann [18]-[20].

\section{LUMPED-DISTRIBUTED NETWORK SYNTHESIS}

In this part, we will apply the invariant subspace theory as developed in Section I, and part of the $L_{2}$ system theory as developed in Section II, to the problem of finding network realizations for a scattering matrix $S$ and a transfer scattering matrix $\Sigma_{21}$. We will show that synthesis techniques of the Darlington type are available in the case that these scattering matrices are roomy. In contrast to Section II, we will not require that a realization is available, so that our systems inputoutput transfer function may be any $H_{m \times n}^{\infty}$ function. Only those results which relate to an input-output description will be used. In Section III.1, we put the scattering matrix theory in an $L^{2}$ systems context and in Section III.2, we introduce the main systemic properties of the scattering matrix which will be used in the two later sections. In Section III.3, 


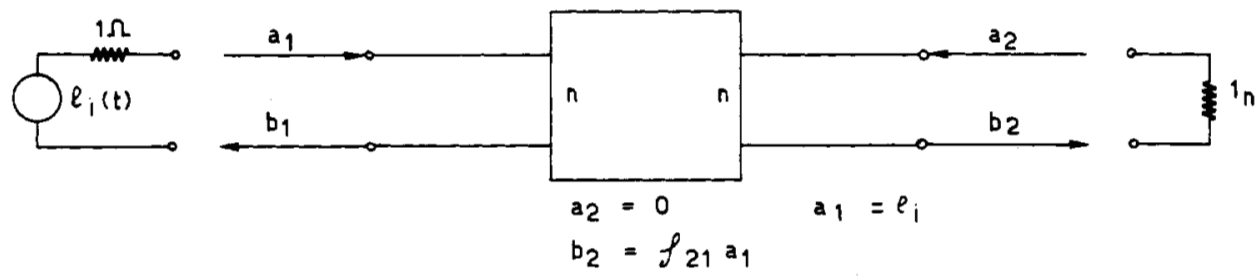

Fig. 1. A transfer scattering matrix.

an Oono-Yasuura type synthesis for roomy scattering matrices is discussed and a key theorem which shows that a roomy scattering matrix can be embedded in a lossless one without alteration of the generalized degree is shown. In Section III.4, we deduce a Darlington type synthesis for a transfer scattering matrix and discuss the practical consequences. The main tool used is coprime factorization. The main results are that one can obtain a spectral factorization, an Oono-Yasuura synthesis, a Belevitch synthesis or a Darlington synthesis by determining coprime factors.

\section{III.1. Networks, $N$-ports, and System Theory}

Cascade synthesis in the context of network theory, always refers to synthesis of so-called $n$-ports. Let $v_{i}(t)$ and $i_{i}(t)$ be the voltage and current respectively for the $i^{\text {th }}$ port of the network. These are real-time functions, but for use in later developments we will allow $v_{i}(t)$ and $i_{i}(t)$ to be complex. The energy absorbed by the network in an interval $\left(t_{1}, t_{2}\right)$ is given by

$$
\varepsilon\left(t_{1}, t_{2}\right)=\int_{t_{1}}^{t_{2}} \operatorname{Re}(\tilde{i} v) d t
$$

whereby $\tilde{i}$ is the Hermitian conjugate of the vector $i$, and " $R \mathrm{e}$ " denotes real part.

We will assume a "creation" time $T=-\infty$ for the network and restrict all inputs to belong to some $L^{2}$ space. We will say that the network is passive if $\&(T, t) \geqslant 0$ for any $t \geqslant T$ and any possible pair of (physical) input vectors $v(t), i(t)$.

Suppose a "physical" voltage source is connected to each port consisting of an "ideal" source $e_{i}(t)$ and a series resistor of $1 \Omega$. Then, defining $a=(v+i) / 2$ and $b=(v-i) / 2$, we have that

$$
\&\left(t_{1}, t_{2}\right)=\int_{t_{1}}^{t_{2}}[\tilde{a} a-\tilde{b} b] d t
$$

whereby $a$ depends only on the source voltage. The first term in (III.1.2), $\int_{t_{1}}^{t_{2}} \tilde{a} a d t$, is obviously the maximal amount of energy the given sources can possibly deliver to any $n$-port connected to them. It is physically clear that, once we have connected the sources, and hence assigned an $a(t)$, any real network will respond with a certain $v(t)$ and $i(t)$ and thus with a $b(t)$. Therefore, any physical network defines a map

$$
a(t) \longmapsto b(t) \text {. }
$$

Moreover if the network is passive, we will always have $\int_{-T}^{\infty} \tilde{b} b d t \leqslant \int_{-T}^{\infty} \tilde{a} a d t$, that is a passive network defines something like a bounded map in some $L^{2}$ space. Thus the mathematical framework:

(i) input space $=$ output space $=L_{n}^{2}(-\infty, \infty)$ with

(ii) The $n$-port defining a map:

$$
\delta: L_{n}^{2}(-\infty, \infty) \longrightarrow L_{n}^{2}(-\infty, \infty) .
$$

The assumption about quadratically bounded response to a quadratically bounded input is justified by our interest in passive synthesis. The variety of networks the framework will produce justifies this limitation.

From now on all maps $\delta$ defined by networks will be linear time invariant (i.e., $\delta \sigma_{\tau}=\sigma_{\tau} \delta$ ), bounded and causal (i.e., $\left.\delta L_{n}^{2}(0, \infty) \subset L_{m}^{2}(0, \infty)\right)$. Such a network is passive if and only if the norm $\|\delta\| \leqslant 1$.

Quite often one is not interested in synthesizing a whole $\delta$ but only part of it. The situation is depicted in Fig. 1, where we have split the ports themselves into $n$ input ports and $m$ output ports. Let $v_{1}, i_{1}$ denote input voltage and current vectors, and $v_{2}, i_{2}$ output voltage and current vectors. Likewise, if we terminate the output with unit resistors and apply inputs as before we have

$$
\begin{aligned}
& a_{1}=\frac{1}{2}\left(v_{1}+i_{1}\right)=\frac{1}{2} e_{1} \\
& a_{2}=\frac{1}{2}\left(v_{2}+i_{2}\right)=0 \\
& b_{2}=\frac{1}{2}\left(v_{2}-i_{2}\right)=v_{2} .
\end{aligned}
$$

So that the relationship between output voltage and source voltage is given by the map

$$
\begin{aligned}
\delta_{21}: L_{n}^{2}(-\infty, \infty) & \longrightarrow L_{m}^{2}(-\infty, \infty) \\
a_{1} & \longmapsto b_{2}
\end{aligned}
$$

which we will require to be linear time invariant bounded and causal. If the network that produces $\delta_{21}$ is passive, clearly $\left\|\dot{\delta}_{21}\right\| \leqslant 1$. A very common way to realize $\delta_{21}$ in electrical engineering, is to make it a part of a network which is lossless (Fig. 1). (Only the case $m=n$ is physically interesting in this case, see e.g. [9], [69]. Thus

$$
\left.\delta=\left[\begin{array}{ll}
\hat{\delta}_{11} & \overbrace{12}^{n} \\
\delta_{21} & \delta_{22}
\end{array}\right]\right) n
$$

where $\delta$ is lossless, i.e., for all inputs $a$, we have as much energy in the output as in the input

$$
\|S a\|=\|a\| \text {. }
$$

This means (with $\delta^{*}$ the Hilbert space adjoint of $\delta$ ) that

$$
\delta^{*} \mathcal{S}=\text { identity. }
$$

Such an $\delta$ will be called $a$ lossless embedding for $\delta_{21}$. Necessary and sufficient conditions for this to happen have been derived by Dewilde [10], Arov [70], Douglas and Helton [25]. Once a lossless embedding is obtained, it is known that the resulting $\delta$ can be, in a sense to be described later, synthesized as a cascade of lossless sections, thereby generalizing the Belevitch synthesis [71], which itself is a generalization of the synthesis of Darlington [72]. Also, the techniques used in the sequel allow a straightforward deduction of a synthesis akin to and more general than the Oono-Yasuura synthesis [73]. 
The operators $\delta$ and $\delta_{21}$ just described will be commonly called scattering operators (for obvious physical reasons) if they are contractive (i.e., if $\|\delta\| \leqslant 1$ or $\left\|\delta_{21}\right\| \leqslant 1$ ). Their characterization in the frequency domain is well known (see also Section II.3):

$$
\begin{aligned}
& \hat{\delta}: L_{n}^{2}(-j \infty, j \infty) \longrightarrow L_{m}^{2}(-j \infty, j \infty) \\
& A(j \omega) \longmapsto B(j \omega)=(\mathfrak{F} b)(j \omega)=S(j \omega)(\mathcal{F} a)(j \omega) \\
&=S(j \omega) A(j \omega)
\end{aligned}
$$

by the Bochner-Chandrasekharan theorem [38], where $S \in H_{m \times n}^{\infty} . S$ will be called the scattering matrix.

\section{III.2. Systems Theory of a Scattering Matrix}

The main purpose of this subsection is to produce a system theoretic description of what we will call the natural state space of the scattering matrix $S$, and to establish the connection with Section II. The assumption on the scattering operator $\delta$, imply that it can be considered as an input-output map in the class described in Section II.3 (i.e., (II.3.1)). Therefore, all the results of Section II.3 apply here. We collect the most important ones for the synthesis problem. The kernel of the Hankel operator associated with $S$

$$
\begin{aligned}
& H_{S}: H_{n}^{2} \longrightarrow H_{m}^{2} \\
& \left(H_{S} A\right)(j \omega)=P_{H_{m}^{2}} S(j \omega) A(-j \omega)
\end{aligned}
$$

is $\subset H_{n}^{2}$. It will be called the nullspace of $S$ in the sequel. It is a right invariant subspace. Its complement $\hat{n}^{\perp}$, as we saw in II.3 is quite a natural state space for the system induced by $\mathfrak{S}$. The importance of transfer functions which have meromorphic pseudo-continuations of bounded type in OLP was discussed in detail in Section II.4. Scattering matrices with this property are also extremely important for synthesis and have been analyzed in [7], [10], [11], [70], [25]. We call them strictly noncyclic or roomy (Section II.4). From Theorem II.4.1 we have the following very important factorizations for such scattering matrices

$$
\begin{aligned}
& S(j \omega)=\Delta_{r}(-j \omega) U_{r}^{-1}(-j \omega) \\
& S(j \omega)=U_{l}^{-1}(-j \omega) \Delta_{l}(-j \omega)
\end{aligned}
$$

where the first is the right coprime factorization, and the second the left coprime factorization and $U_{r}, U_{l}$ are lossless in $H_{n \times n}^{\infty}$ and $H_{m \times m}^{\infty}$ respectively, and characterize the state spaces of the two canonically constructed realizations in Section II.4. In particular

$$
\hat{\text { 负 }}=U_{r} H_{n}^{2} \text {. }
$$

Theorem III.2.1: Let $S(j \omega)$ be roomy and consider the factorizations (III.2.2). Then $\operatorname{det} U_{r}=\operatorname{det} U_{l}$.

Proof: An elementary proof is given in [11], [12]. A proof based on the Nordgren theory is in [22].

This theorem is crucial for two reasons: 1 ) because it allows an extension of the finite dimensional degree theory to infinitedimensional systems and networks and 2) because it allows for practical embedding algorithms. The first point will be discussed to close this section while the second will be in the next subsection.

We will now develop our degree theory for an $m \times n$ roomy system. Since all information about the natural state space of $S$ is contained in the matrix $U_{r}$ (or $U_{l}$ ) it will be enough to develop a degree theory for $U_{r}$ and we will identify the degree of $U_{r}$ with the degree of $\mathcal{S}$. For a lossless matrix function $U$ a ,ood measure for the complexity of $U$ is obtained by consider- ing det $U$. To see that, let $\mathfrak{I}_{n}$ be the multiplicative monoids of $n \times n$ inner matrix functions. We will call $\delta: \mathcal{T}_{n} \rightarrow \mathcal{T}_{1}$ a degree map if the following conditions are satisfied:

(i) $\delta: \mathcal{T}_{n} \longrightarrow \mathcal{T}_{1}$ and $\delta\left(1_{n}\right)=1$

(ii) if $U=U_{1} U_{2} ; U_{1}, U_{2} \in \mathcal{T}_{n}$ then $\delta(U)=\delta\left(U_{1}\right) \cdot \delta\left(U_{2}\right)$

(iii) $\delta\left(\left[\begin{array}{ll}\varphi & 0 \\ 0 & 1_{n-1}\end{array}\right]\right)=\varphi$

where $\varphi$ is a lossless scalar $\left(\varphi \in \mathfrak{T}_{1}\right)$ and $1_{n-1}$ the unit $n-1$ matrix. The main property is (ii) which says that $\delta$ is a monoid homomorphism. It is not difficult to prove [11] that det $U=\delta(U)$ (Note that this does not work for a general $n \times n$ matrix $A$ !). It is well known in classical network theory [69] that the degree of a finite (rational) lossless matrix is equal to the degree of its determinant. The degree map extends this property to roomy matrix functions.

For synthesis purposes, it is necessary to look a bit more closely to the analytic structure of $U$ and its determinant $\varphi$. Typical lossless matrices and functions are as follows.

1) So called Blaschke factors:

$$
U=1_{n}-\frac{2 \alpha_{0} u \tilde{u}}{p+\tilde{p}_{0}} \quad \varphi=\frac{p-p_{0}}{p+\tilde{p}_{0}}
$$

where $\alpha_{0}=\operatorname{Re} p_{0}>0, u$ is an $n$-vector with unit norm $(\tilde{u} u=1)$ and $p_{0}$ is a zero in the ORP.

2) So called singular factors:

$$
U=e^{-\gamma u \tilde{u}} \quad \varphi=e^{-\gamma}
$$

with either $\gamma=k p$ or

$$
\gamma=\frac{k\left(1-p \cdot j \omega_{0}\right)}{p-j \omega_{0}}
$$

$u$ an $n$-vector with $\tilde{u} u=1, j \omega_{0}$ a point on the imaginary axis, and $k>0$ a positive constant. The theory of Riesz-HerglotzPotapov [31], [32], [76] asserts that any lossless matrix $U$ and its determinant $\varphi$ can be viewed as the limit of a sequence of products of the two types. More precisely any $U$ decomposes as

$$
U=U^{B} \cdot U^{S} \quad \varphi=\varphi^{B} \cdot \varphi^{S}
$$

where $U^{B}$ is an (eventually infinite) product of Blaschke factors, $\varphi^{B}=\operatorname{det} U^{B}$, and all poles in the OLP (zeros in the ORP) are represented according to their multiplicity; $U^{S}$ is a product integral of elementary factors (limit of a finite product of elementary factors in the sense of [32]) and

$$
\varphi^{S}=\operatorname{det} U^{S}=e^{-p r} \exp \left[-\int_{-\infty}^{\infty} \frac{t p+j}{t+j p} d \mu(t)\right]
$$

where $\mu(t)$ is a finite singular measure on the $j \omega$-axis. Note that $\varphi^{S}$ is analytic wherever $U^{S}$ is and conversely.

\section{III.3. Scattering Matrix Synthesis Through Lossless Embedding}

In this subsection, we will attack the synthesis problem from an "Oono-Yasuura" point of view, i.e., we will try to embed a given $n \times n$ scattering matrix $S(j \omega)$ in a $2 n \times 2 n$ lossless one, and then synthesize the lossless $\Sigma$ by means of a RieszHerglotz-Potapov factorization. The ability to embed $S$ in a unitary $\Sigma$ is dependent upon the accomplishment of two steps: (i) First an analytic $\Sigma_{21}$ has to be determined so that $\tilde{\Sigma}_{21} \Sigma_{21}=1_{n}-\tilde{S} S$ (spectral factorization). (ii) Second the 
matrix

$$
\left[\begin{array}{c}
S \\
\Sigma_{21}
\end{array}\right]
$$

has to be embedded in a unitary (lossless) one. Moreover operations (i) and (ii) have to be executed in a minimal fashion, i.e., without increasing the degree of the system.

It is a well-known fact in the theory of multiplicative operators (for an excellent discussion, see, e.g., [26, ch. 10]), that step (i) can always be executed. This point has also been extensively discussed in the theory of resolution spaces [83], [84]. The proof of this is usually "existential," i.e., it shows the existence of a spectral factor $\Sigma_{21}$, but does not give an algorithm to actually compute the factor. We will accept the existential statement here and show how to deal with the spectral factorization in the next subsection.

In this subsection, we will concentrate on point (ii) and show that our coprime factorization theory provides an embedding if and only if $S$ is roomy.

Also, we will indicate how coprime factorization potentially yields an algorithm to compute the embedding. First a few observations about $\Sigma_{21}$.

Let

$$
\tilde{\Sigma}_{21} \Sigma_{21}=1_{n}-\tilde{S} S
$$

be a spectral factorization for $1_{n}-\tilde{S} S$. We can always suppose that $\Sigma_{21}$ is outer, for any inner factor in $\Sigma_{21}$ cancels, in the first member of (III.3.2).

Now, let $S$ be an $n \times n$, not identically singular scattering matrix, and let $\Sigma_{21}$ be an outer spectral factor, as given by (III.3.2). Let $\hat{n}_{1}$ be the nullspace of $S$ as defined in the previous section.

Theorem III.3.1: The nullspace $\hat{h}_{1}$ of $S$ lays in the nullspace $\hat{\mathrm{h}}_{1}^{\prime}$ of $\Sigma_{21}$. The two coincide if and only if $S$ itself is outer.

Proof: We have $F \in \hat{A}_{1}$ if and only if $S(-j \omega) F$ is analytic. But if $S(-j \omega) F$ is analytic, then, by (III.3.2), so is $\tilde{\Sigma}_{21}(-j \omega) \Sigma_{21}(-j \omega) F$. This implies that $\Sigma_{21}(-j \omega) F$ is also analytic. For, suppose not, then there is $G_{1}$ conjugate analytic, so that $\widetilde{\Sigma}_{21}(-j \omega) G_{1}$ is analytic. It follows that $G_{1}(-j \omega) \perp \Sigma_{21} H_{n}^{2}$ and, since $\Sigma_{21}$ is outer and $G_{1}(-j \omega)$ analytic, $G_{1}=0$. This shows $\hat{M}_{1} \subset \hat{M}_{1}^{\prime}$. If $S$ is outer, then also 般 ${ }_{1} \subset \hat{N}_{1}$.

Q.E.D.

It follows that $\hat{h}_{1}$ is the nullspace of the matrix (III.3.1). Suppose now that $S$ is roomy, then we have shown that the generalized degree of (III.3.1) is equal to the generalized degree of $S$. A right coprime factorization for (III.3.1) gives

$$
\left[\begin{array}{c}
S \\
\Sigma_{21}
\end{array}\right]=\sigma_{-} \Delta_{r} \cdot \sigma_{-} U_{r}^{-1}
$$

and (III.3.3) is of course roomy together with $S$.

Theorem III.3.2: The left coprime factorization (up to a trivial constant unitary factor) for (III.3.3) is given by

$$
\left[\begin{array}{c}
S \\
\Sigma_{21}
\end{array}\right]=\Sigma \cdot\left[\begin{array}{l}
1_{n} \\
0_{n}
\end{array}\right]
$$

where $\Sigma$ is the minimal lossless embedding for $S$ with outer $\Sigma_{21}$. It follows that $\Sigma$ has the same generalized degree as $S$.

Proof: Let

$$
\Sigma \cdot\left[\begin{array}{l}
A_{11} \\
A_{21}
\end{array}\right]
$$

be a left coprime factorization for

$$
\left[\begin{array}{c}
S \\
\Sigma_{21}
\end{array}\right]
$$

We have, because of (III.3.2) that $\tilde{A}_{11} A_{11}+\tilde{A}_{21} A_{21}=1_{n}$. It should be remarked that the left coprime factorization originates in the dual system (see Section II.4). Translating the notation used there we have

$$
\begin{aligned}
& \sigma_{-} U_{l}^{-1} \longleftrightarrow \Sigma \\
& \sigma_{-} \Delta_{l} \longleftrightarrow\left[\begin{array}{l}
A_{11} \\
A_{21}
\end{array}\right] .
\end{aligned}
$$

Hence

$$
\left[\begin{array}{l}
A_{11} \\
A_{21}
\end{array}\right]
$$

has to be considered conjugate analytic (we are proving it is actually constant). We thus have that $\sigma_{-} \widetilde{\Sigma}$ and

$$
\sigma_{-}\left[\begin{array}{l}
A_{11} \\
A_{21}
\end{array}\right]
$$

are left coprime and thus there are sequences $M_{i} \in H_{n \times 2 n}^{\infty}$ and $N_{i} \in H_{2 n \times 2 n}^{\infty}$ such that

$$
\lim _{i \rightarrow \infty}\left\{\sigma_{-}\left[\begin{array}{l}
A_{11} \\
A_{21}
\end{array}\right] M_{i}+\sigma_{-} \tilde{\Sigma} N_{i}\right\}=1_{2 n}
$$

(the limit representing one or the other type of convergence mentioned before: see Section I).

Premultiplying with $\left[\tilde{A}_{11}, \tilde{A}_{21}\right]$ gives

$$
\begin{aligned}
{\left[\tilde{A}_{11}, \tilde{A}_{21}\right] } & =\lim _{i \rightarrow \infty}\left\{\sigma_{-} M_{i}+\left[\tilde{A}_{11}, \tilde{A}_{21}\right] \Sigma \sigma_{-} N_{i}\right\} \\
& =\lim _{i \rightarrow \infty}\left\{\sigma_{-} M_{i}+\left[\tilde{S}, \tilde{\Sigma}_{21}\right] \sigma_{-} N_{i}\right\} .
\end{aligned}
$$

The right-hand side is by necessity conjugate analytic, and so is thus the left-hand side. The result is that

$$
\left[\begin{array}{l}
A_{11} \\
A_{21}
\end{array}\right]
$$

is a matrix which is both analytic and conjugate analytic, hence constant.

Moreover, since (III.3.3) and (III.3.4) are coprime factorizations for

$$
\left[\begin{array}{c}
S \\
\Sigma_{21}
\end{array}\right]
$$

we have by Theorem III.2.1, that $\operatorname{det} U_{r}=\operatorname{det} \Sigma$ and hence the embedding of $S$ has the same degree as $U_{r}$, which is the degree of $S$. This proves the theorem.

Because of Theorem III.3.2 it follows that an embedding of

$$
\left[\begin{array}{c}
S \\
\Sigma_{21}
\end{array}\right]
$$

reduces to a simple coprime factorization. This result provides for an alternative approach to the (viery difficult) OonoYasuura [73] synthesis technique and in fact, produces both an alternative proof for their result and a synthesis algorithm, the main lines of which we want to discuss now. 


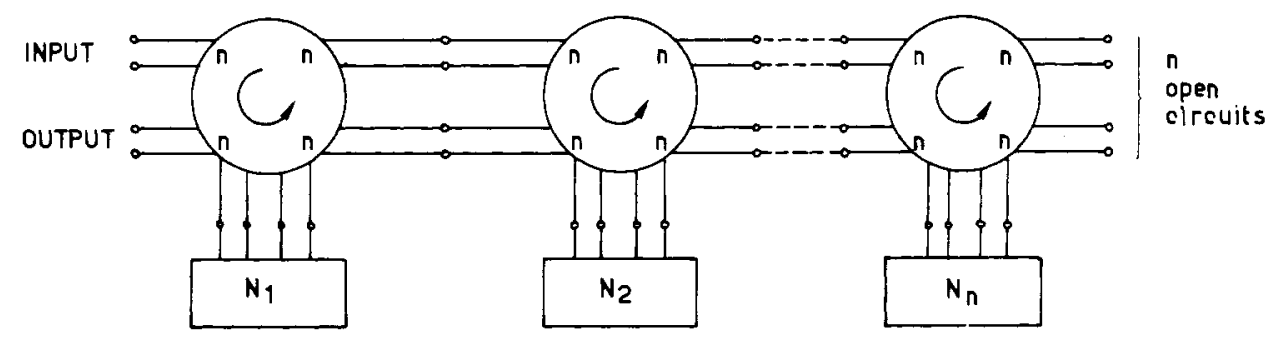

Fig. 2. The network resulting from a factorization of $\Sigma$.

When

$$
\left[\begin{array}{c}
S \\
\Sigma_{21}
\end{array}\right]
$$

is rational, then it can have only a finite number of poles in OLP. A Blaschke factor,

$$
1_{2 n}-\frac{2 \alpha_{0} u \tilde{u}}{p+\tilde{p}_{0}}
$$

can be extracted from it, with reduction of degree if [9], [13]:

1) $-\widetilde{p}_{0}$ is a pole of $S$;

2) the vector $u$ is chosen according to the following rule: suppose

$$
\left[\begin{array}{c}
S \\
\Sigma_{21}
\end{array}\right]=\sum_{i=l}^{1} \frac{A_{i}}{\left(p+\tilde{p}_{0}\right)^{i}}+\cdots
$$

is a Laurent expansion at $-\widetilde{p}_{0}$, and

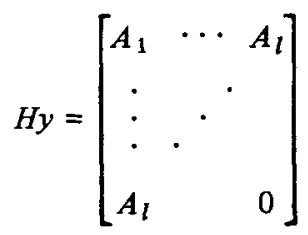

is a Hankel matrix based on the sequence $A_{1}, \cdots, A_{l}, 0, \cdots$ then the vector $[u, 0, \cdots, 0]^{T}$ is in the columnspace of $H$, or else, there is a $2 n l$ vector $y$ such that

$$
H y=\left[\begin{array}{c}
u \\
0 \\
\vdots \\
0
\end{array}\right] .
$$

We can hence recursively determine a sequence of Blaschke factors $B_{1}, B_{2}, \cdots, B_{\delta}$ ( $\delta$ being the degree of $S$ ) until we terminate in a constant matrix, so that

$$
\left[\begin{array}{c}
S \\
\Sigma_{21}
\end{array}\right]=B_{1} \cdot B_{2} \cdots B_{\delta} \cdot\left[\begin{array}{c}
A_{11} \\
A_{21}
\end{array}\right] .
$$

It suffices to represent

$$
\left[\begin{array}{l}
A_{11} \\
A_{21}
\end{array}\right]=A\left[\begin{array}{l}
1_{n} \\
0_{n}
\end{array}\right]
$$

with $A$ constant unitary and the embedding (together with a factorization) is given by

$$
\Sigma=B_{1} \cdot B_{2} \cdots B_{\delta} \cdot A
$$

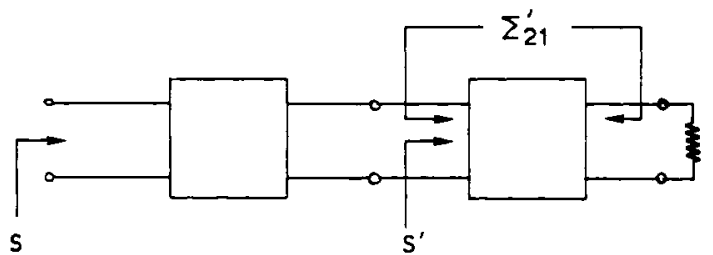

Fig. 3. Two cascaded sections.

Network realizations for (III.3.12) are well known [10], and result in the structure pictured in Fig. 2. It should be noted that this structure is impractical since it is not a cascade realization for $S$ in the true sense. For that reason, we will not pause any longer on this case and only indicate how the theory generalizes for infinite structures. In the next section, the cascade case will be discussed.

Suppose now that $S$ is roomy (but not identically singular). $\Sigma$ then consists of a Blaschke part and a singular part the degree of which is represented by $(\operatorname{det} \Sigma)^{B}$ and $(\operatorname{det} \Sigma)^{S}$. There are good physical reasons to suppose that $S$, and following $\Sigma_{21}$ and $\Sigma$ are meromorphic, this being a result of considerations about Maxwell's equations; and we will restrict the discussions to that case (this restricts the singularities on the $j \omega$-axis to the point $\infty$, but any other point would be treated similarly but would result in nonphysical transmission lines).

By the Riesz-Herglotz-Potapov factorization, we have

$$
\Sigma=\prod_{i=1}^{\infty} B_{i} \cdot \int^{l} e^{-p d M(t)}
$$

where $B_{i}$ are Blaschke factors, $M(t)$ is a monotonically increasing family of nonnegative Hermitian functions and the sign

$$
\int_{0}^{l}
$$

indicates a "product" integral, limit of a product

$$
e^{-p \Delta_{2} M} \cdot e^{-p \Delta_{2} M} \cdots e^{-p \Delta_{n} M}
$$

where $\Delta_{i} M=M\left(t_{i+1}\right)-M\left(t_{i}\right)$, the limiting procedure is to be seen as a Stieltjes integral, and $\operatorname{tr}[M(t)]=t$. Following Belevitch [71], it can easily be seen that the network resulting from this product decomposition is of the type depicted in Fig. 2, $N_{j}$ standing for either a first-or second-degree network, a transmission line, or an elementary section of a transmission line, and the circulator being the circuit element which realizes the factor decomposition. In circuit theoretical practice one is usually asked to synthesize a transfer scattering matrix $\Sigma_{21}$ and not a scattering matrix $S$, and we will devote the next 
Subsection to that case. Also, in the next Subsection we will indicate a method to obtain the spectral factor by coprime factorization.

\section{III.4 Transfer Scattering Matrix Synthesis and Determination of the Spectral Factor}

In this subsection, we will first discuss methods to determine, by coprime factorization, the spectral factor $\Sigma_{21}$ out of $S$ for a roomy system. The idea behind the method is closely related to the idea of cascade synthesis. First, suppose a lossless realization has been found for $S$, terminated in $n$ unit resistors. The $\Sigma_{21}$ of the realization (which is called the transfer scattering matrix) is related to $S$ by

$$
\tilde{\Sigma}_{21} \Sigma_{21}=1-\tilde{S} S
$$

and is thus a spectral factor for $1-\tilde{S} S$.

Next, suppose we have a cascade realization for $S$, consisting of two cascaded sections (Fig. 3 ). Then $S$ is uniquely determined by $N$ and $S^{\prime}$ and not by the $\Sigma_{21}^{\prime}$ in the section $N^{\prime}$. Hence, if we succeed in providing a recursive method for the extraction of lossless cascade sections out of $S$, and this should be possible without previous knowledge of $\Sigma_{21}$, then $\Sigma_{21}$ will be obtained as the global transfer scattering matrix of the cascade. This procedure, in network terms, is known as the Belevitch synthesis [69], [71], and we will attack it from a different point of view, namely coprime factorization. A cascade synthesis for $\Sigma$ (Fig. 3) coincides with a factorization of the transmission matrix $\Theta$ given by:

$$
\left[\begin{array}{l}
A_{1} \\
B_{1}
\end{array}\right]=\Theta\left[\begin{array}{l}
B_{2} \\
A_{2}
\end{array}\right] \quad \Theta=\left[\begin{array}{cc}
\Sigma_{21}^{-1} & -\Sigma_{21}^{-1} \Sigma_{22} \\
\Sigma_{11} \Sigma_{21}^{-1} & \Sigma_{12}-\Sigma_{11} \Sigma_{21}^{-1} \Sigma_{22}
\end{array}\right]
$$

or, with $P=1_{n} \dot{+} 0_{n}, P^{\perp}=0_{n}+1_{n}$ and

$$
\begin{gathered}
\Delta=\left[\begin{array}{cc}
0 & 1_{n} \\
1_{n} & 0
\end{array}\right] \\
\Theta=\left(P+P^{\perp} \Delta \Sigma\right)\left(P \Delta \Sigma+P^{\perp}\right)^{-1} .
\end{gathered}
$$

Our problem can thus be formulated as follows: find a "minimal" (i.e., of least degree) factorization of $\Theta$ from $S$ without previous knowledge of $\Sigma_{21}$. There is an additional difficulty here which we did not encounter in Section III.3: $\Sigma_{21}^{-1}$, and hence $\Theta$ is not necessarily analytic in the sense defined before and moreover, $\Theta$ need not belong to $H_{2 n}^{\infty} \times 2 n$. Our coprime factorization theory was explicitely stated in the context of $H^{\infty}$ theory and we will have to extend it. The characteristic property of $\Theta$, corresponding to the property that $\Sigma$ is a scattering matrix, is the following (fix $p \in O R P$ and let ${ }_{\text {indicate Hermitian conjugation). Let }}$

$$
J=\left[\begin{array}{cc}
1_{n} & 0 \\
0 & -1_{n}
\end{array}\right]
$$

then

$$
\tilde{\Theta} J \Theta-J=\left(\tilde{\Sigma} \Delta P+P^{\perp}\right)^{-1}\left(1_{2 n}-\tilde{\Sigma} \Sigma\right)\left(P \Delta \Sigma+P^{\perp}\right)^{-1}
$$

so that, in the ORP we have that

$$
\tilde{\Theta} J \Theta-J \geqslant 0
$$

or $[32] \Theta$ is $J$ expansive in the ORP.
Moreover, if $\Sigma$ is lossless, and hence unitary a.e. on the imaginary axis, we have that $\widetilde{\Theta} J \Theta-J=0$ a.e. on the imaginary axis. For lack of a better name, we will call a $\Theta$ deduced from an analytic scattering matrix $\Sigma$, passive and one deduced from a conjugate analytic one, antipassive. As in Section III.3, it will not be necessary to work on the total matrix $\Theta$ but only on matrices of the type:

$$
\Theta=\left[\begin{array}{c}
\Sigma_{21}^{-1} \\
S \Sigma_{21}^{-1}
\end{array}\right]
$$

which we will call passive (or antipassive) according to whether

$$
\left[\begin{array}{c}
S \\
\Sigma_{21}
\end{array}\right]
$$

may be considered a contractive analytic (or conjugate analytic) matrix. As is the case for lossless matrices, there is a partial ordering available for $J$-unitary matrices, so that a $J$ unitary, passive $\Theta_{1}$ is a right multiple of a $J$-unitary $\Theta_{2}$ if and only if $\widetilde{\Theta}_{1} J \Theta_{1} \geqslant \widetilde{\Theta}_{2} J \Theta_{2}$ a.e. in the ORP, for we have, with $\Theta_{1}=\Theta_{3} \Theta_{2}$, that $\tilde{\Theta}_{1} J \Theta_{1}-J=\widetilde{\Theta}_{2}\left(\widetilde{\Theta}_{3} J \Theta_{3}-J\right) \Theta_{2}+\left(\widetilde{\Theta}_{2} J \Theta_{2}-\right.$ $J$ so that $\widetilde{\Theta}_{3} J \Theta_{3}-J \geqslant 0$ in the ORP if $\widetilde{\Theta}_{1} J \Theta_{1} \geqslant \widetilde{\Theta}_{2} J \Theta_{2}$ in the ORP. Also, if $\Theta_{1}$ and $\Theta_{2}$ are $J$-unitary and passive, and $\tilde{\Theta}_{1} J \Theta_{1}=\tilde{\Theta}_{2} J \Theta_{2}$ a.e. in the ORP, then $\Theta_{1}=\Theta_{2}$, for if $\Theta_{3}=$ $\Theta_{1} \Theta_{2}^{-1}$ is such that $\tilde{\Theta}_{3} J \Theta_{3}-J=0_{2 n}$ a.e. ORP then its corresponding $\Sigma_{3}$ has the property that $\tilde{\Sigma}_{3} \Sigma_{3}-1_{2 n}=0_{2 n}$ a.e. in the ORP and hence is constant.

Given a $2 n \times n$ matrix $\left[\begin{array}{ll}A_{11}^{T} & A_{21}^{T}\end{array}\right]^{T}$ which has the property that

$$
\Theta^{-1}\left[\begin{array}{l}
A_{11} \\
A_{21}
\end{array}\right]=\left[\begin{array}{l}
A_{11}^{\prime} \\
A_{21}^{\prime}
\end{array}\right]
$$

is antipassive, it makes sense, as with the previous coprime factorization, to wonder whether there is a "smallest $\Theta$ " with that property. In other words, whether there is a coprime factorization for

$$
\left[\begin{array}{l}
A_{11} \\
A_{21}
\end{array}\right] \text { as } \Theta\left[\begin{array}{l}
A_{11}^{\prime} \\
A_{21}^{\prime}
\end{array}\right], \Theta J \text {-unitary and }\left[\begin{array}{l}
A_{11}^{\prime} \\
A_{21}^{\prime}
\end{array}\right] \text { antipassive. }
$$

An application of Zorn's lemma reduces the proof of the property to: let $\Theta_{1}$ and $\Theta_{2}$ be as in (III.4.9), then so is their greatest common left $J$-unitary divisor. We will not need the property in this generality, although we conjecture it to be true. We will say that $\Theta$ is the left J-unitary cofactor of

$$
\left[\begin{array}{l}
A_{11} \\
A_{21}
\end{array}\right]
$$

if indeed it is the unique passive $J$-unitary factor (unique except for a trivial right constant $J$-unitary factor of course) which left divides any $J$-unitary passive factor $\Theta_{1}$ for which

$$
\Theta_{2}^{-1}\left[\begin{array}{l}
A_{11} \\
A_{21}
\end{array}\right]
$$

is antipassive. The basis of our algorithm is then given by:

Theorem III.4.1: Given an $n \times n$ scattering matrix $S$, then the left $J$-unitary cofactor for

$$
\left[\begin{array}{c}
\left(1_{n}-\tilde{S} S\right)^{-1} \\
S\left(1_{n}-\tilde{S} S\right)^{-1}
\end{array}\right]
$$


is the matrix $\Theta$ whose corresponding $\Sigma$ is a minimal lossless embedding for $S$.

Proof: We have

$$
\left[\begin{array}{l}
A_{1} \\
A_{2}
\end{array}\right] \triangleq\left[\begin{array}{c}
\left(1_{n}-\tilde{S} S\right)^{-1} \\
S\left(1_{n}-\tilde{S} S\right)^{-1}
\end{array}\right]=\left[\begin{array}{c}
\Sigma_{21}^{-1} \\
S \Sigma_{21}^{-1}
\end{array}\right] \tilde{\Sigma}_{21}^{-1}
$$

where $\Sigma_{21}$ is the outer cofactor for $S$. It is clear that

$$
\Theta^{-1}\left[\begin{array}{l}
A_{1} \\
A_{2}
\end{array}\right]=\left[\begin{array}{c}
\tilde{\Sigma}_{21}^{-1} \\
0_{n}
\end{array}\right]
$$

is antipassive so that the $\Theta$ obtained from the minimal embedding with outer $\Sigma_{21}$ satisfies the requirements for being a factor. It will be the cofactor if we proof that, for $\Theta_{1}$ such that

$$
\Theta_{1}^{-1}\left[\begin{array}{l}
A_{1} \\
A_{2}
\end{array}\right]=\left[\begin{array}{l}
A_{1}^{\prime} \\
A_{2}^{\prime}
\end{array}\right]
$$

is antipassive and whose corresponding $\left(\Sigma_{1}\right)_{21}$ is outer, then there is a $J$-unitary passive $\Theta_{3}$ such that

$$
\Theta_{1}=\Theta \cdot \Theta_{3} \text {. }
$$

Of course, $\Theta_{3}=\Theta^{-1} \Theta_{1}$ is well defined, the question being whether it is passive. Clearly we have

$$
\left[\begin{array}{c}
A_{1}^{\prime} \\
A_{2}^{\prime}
\end{array}\right]=\Theta_{3}^{-1}\left[\begin{array}{c}
\tilde{\Sigma}_{21}^{-1} \\
0_{n}
\end{array}\right]=J \tilde{\Theta}_{3} J\left[\begin{array}{c}
\tilde{\Sigma}_{21}^{-1} \\
0_{n}
\end{array}\right]
$$

so that, denoting

$$
\Theta_{3}=\left[\begin{array}{ll}
\Theta_{11} & \Theta_{12} \\
\Theta_{21} & \Theta_{22}
\end{array}\right]
$$

and the corresponding unitary

$$
\Sigma_{3}=\left[\begin{array}{ll}
\sigma_{11} & \sigma_{12} \\
\sigma_{21} & \sigma_{22}
\end{array}\right]
$$

we have

$$
\left[\begin{array}{l}
A_{1}^{\prime} \\
A_{2}^{\prime}
\end{array}\right]=\left[\begin{array}{ll}
\widetilde{\sigma}_{21}^{-1} & \tilde{\Sigma}_{21}^{-1} \\
\widetilde{\sigma}_{22} & \tilde{\sigma}_{21}^{-1} \widetilde{\Sigma}_{21}^{-1}
\end{array}\right] .
$$

Since (III.4.14) is supposed to be antipassive, it follows that $\sigma_{21} \Sigma_{21}$ has to be analytic. Since $\Sigma_{21}$ is outer, $\sigma_{21}$ has to be analytic as well. Also, $\sigma_{22}$ has to be analytic as a product of two analytic functions. From the fact that $\Theta_{1}=\Theta_{3}$, we deduce:

$$
\Sigma_{1}=\left[\begin{array}{c}
\Sigma_{11}+\Sigma_{12} \sigma_{11}\left(1_{n}-\Sigma_{22} \sigma_{11}\right)^{-1} \Sigma_{21} \\
\Sigma_{12}\left(1_{n}-\sigma_{11} \Sigma_{22}\right)^{-1} \sigma_{12} \\
\sigma_{21}\left(1_{n}-\Sigma_{22} \sigma_{11}\right)^{-1} \Sigma_{21} \\
\sigma_{22}+\sigma_{21} \Sigma_{22}\left(1_{n}-\sigma_{11} \Sigma_{22}\right)^{-1} \sigma_{12}
\end{array}\right]
$$

$\Sigma_{1}$ is analytic and such that $\left(\Sigma_{1}\right)_{21}$ is outer, so that we can deduce that:

i) $\sigma_{21}\left(1_{n}-\Sigma_{22} \sigma_{11}\right)^{-1} \Sigma_{21}$ is analytic and outer;

ii) $\sigma_{21}\left(1_{n}-\Sigma_{22} \sigma_{11}\right)^{-1} \Sigma_{22} \sigma_{12}$ is analytic;

iii) $\Sigma_{12} \sigma_{11}\left(1_{n}-\Sigma_{22} \sigma_{11}\right)^{-1}$ is analytic;

iv) $\Sigma_{12}\left(1_{n}-\sigma_{11} \Sigma_{22}\right)^{-1} \sigma_{12}$ is analytic.

At this point we have to use two properties of outer functions ( $A, B$, and $C$ are $n \times n$ square matrices):

a) Suppose $A=B C$ is analytic and either $B$ or $C$ is outer, then $B$ and $C$ are analytic.
To see this suppose that $B$ is outer. Then, (see Section I) there is a sequence $M_{i}$ of analytic functions with $\lim _{i \rightarrow \infty}$. $M_{i} B=1_{n}$. It follows then $C=\lim _{i \rightarrow \infty} M_{i} A$ must be analytic.

b) Suppose that $B$ and $C$ are analytic matrices and that $B$ and $C$ are outer. Then $B C$ has to be outer. Indeed a matrix $A$ is outer if and only if its determinant is outer.

From i) and b) it follows that $\sigma_{21}\left(1_{n}-\Sigma_{22} \sigma_{11}\right)^{-1}$ is analytic and outer, so that

$$
\sigma_{21}=T\left(1_{n}-\Sigma_{22} \sigma_{11}\right)
$$

with $T$ outer. It follows that $1_{n}-\Sigma_{22} \sigma_{11}$ and also $\Sigma_{22} \sigma_{11}$ are analytic. From (iii) we have that $\Sigma_{12} \sigma_{11}$ is analytic as well. From (ii) $\Sigma_{22} \sigma_{12}$ is analytic because $\sigma_{21}\left(1_{n}-\Sigma_{22} \sigma_{11}\right)^{-1}$ is outer and we have that

$$
\begin{aligned}
\Sigma_{12} \sigma_{12}=\Sigma_{12}\left(1_{n}-\sigma_{11} \Sigma_{22}\right)^{-1} & \sigma_{12} \\
& -\Sigma_{12} \sigma_{11}\left(1_{n}-\Sigma_{22} \sigma_{11}\right)^{-1} \Sigma_{22} \sigma_{12}
\end{aligned}
$$

is analytic because of ii) and iv).

Now, $\Sigma_{12}$ and $\Sigma_{22}$ belong to a minimal embedding of $S$, so they have to be right coprime, for otherwise $\Sigma_{12}=\Sigma_{12}^{\prime} U$, $\Sigma_{22}=\Sigma_{22}^{\prime} U$ with $\Sigma_{12}^{\prime}$ and $\Sigma_{22}^{\prime}$ analytic and

$$
\left[\begin{array}{cc}
S & \Sigma_{12}^{\prime} \\
\Sigma_{21} & \Sigma_{22}^{\prime}
\end{array}\right]
$$

would be a minimal embedding with smaller determinant and hence with smaller (generalized) degree.

Thus, there are $M_{i}$ and $N_{i}$ such that $\lim _{i \rightarrow \infty}\left(M_{i} \Sigma_{12}+N_{i} \Sigma_{22}\right)=$ $1_{n}$ and it follows that

$$
\begin{aligned}
& \sigma_{11}=\lim _{i \rightarrow \infty}\left(M_{i} \Sigma_{12} \sigma_{11}+N_{i} \Sigma_{22} \sigma_{11}\right) \\
& \sigma_{12}=\lim _{i \rightarrow \infty}\left(M_{i} \Sigma_{12} \sigma_{12}+N_{i} \Sigma_{22} \sigma_{12}\right)
\end{aligned}
$$

are analytic. This proves the theorem.

Corollary III.4.2: In order to obtain a spectral factor $\Sigma_{21}$ for $S$ and an embedding, it is enough to produce a coprime factorization for (III.4.10) with a left $J$-unitary cofactor. Note that the existence and uniqueness of the cofactor is ascertained by the theorem.

Theorem III.4.1 solves in principle the synthesis problem for roomy scattering matrices. In practice, however, it does provide for a new algorithm in the rational case, but experiences quite a few difficulties in the nonrational case. We will first discuss how the algorithm goes in the rational case and then proceed to a discussion of practical consequences in the nonrational case.

a) The rational case: We have to produce an outer $\Sigma_{21}$ out of (III.4.10) as requested by the minimality of $\Theta$. Poles of (III.4.10) in the OLP and on the imaginary axis are what is known as transmission zeros. In fact, without loss of generality, we may suppose $S$ outer to start with, in which case the poles of (III.4.10) are exactly transmission zeros (for an extensive discussion of transmission properties, see [9]). Two types of factors can occur, according to whether the transmission zero is in the OLP or on the axis. In the first case it is of type [9]:

$$
1_{2 n}-\frac{2 \alpha_{0} u \tilde{u} J}{p-p_{0}}, \quad \alpha_{0}=\operatorname{Re} p_{0}, \quad \tilde{u} J u=-1, \quad \alpha_{0}<0
$$

for a transmission zero in the OLP and in the second case:

$$
1_{2 n}+\frac{r u \tilde{u} J}{p-j \omega_{0}}, \quad r>0, \quad \tilde{u} J u=0
$$


for a transmission zero on the $j \omega$-axis, with

$$
1_{2 n}+p r u \tilde{u} J, \quad r>0, \quad \tilde{u} J u=0
$$

for the zero at infinity. Type (III.4.18) results in what is known as a Darlington section, (III.4.19) as a Brune section.

From a numerical point of view one has to determine the point $p_{0}$ or $j \omega_{0}$ and the vector $u$ for each section extraction. This is done exactly as described in Section III.3, formulas (III.3.9)-(III.3.11), taking care of the extra conditions in (III.4.18)-(III.4.19). We have for example; with

$$
S=\left[\begin{array}{cc}
\frac{k \cdot p}{p+1} & 0 \\
\frac{k}{p+1} & \frac{p}{(p+2)}
\end{array}\right] k=\frac{2}{\sqrt{5}}
$$

that

$$
\begin{aligned}
& {\left[\begin{array}{l}
\left(1-S^{*} S\right)^{-1} \\
S\left(1-S^{*} S\right)^{-1}
\end{array}\right]} \\
& {\left[\begin{array}{ll}
-5 p^{2}+5 & -\frac{\sqrt{5}}{2} p^{3}+\frac{\sqrt{5}}{2} p^{2}+\sqrt{5} p \\
\frac{\sqrt{5}}{2} p^{3}+\frac{\sqrt{5}}{2} p^{2}-\sqrt{5} p & \frac{1}{4} p^{4}-\frac{5}{4} p^{2}+1 \\
-2 \sqrt{5} p^{2}+2 \sqrt{5} p & -p^{3}+2 p^{2} \\
\frac{\sqrt{5}}{2} p^{3}-\frac{\sqrt{5}}{2} p^{2}-2 \sqrt{5} p+2 \sqrt{5} & \frac{1}{4} p^{4}-\frac{1}{2} p^{3}-\frac{5}{4} p^{2}+\frac{5}{2} p
\end{array}\right]}
\end{aligned}
$$

so that there is a transmission zero of degree two at infinity Extracting two Brune sections produces:

$$
\begin{aligned}
& \Theta=\left[\begin{array}{cccc}
1 & 0 & 0 & 0 \\
0 & 1+\frac{p}{2} & 0 & -\frac{p}{2} \\
0 & 0 & 1 & 0 \\
0 & \frac{p}{2} & 0 & 1-\frac{p}{2}
\end{array}\right]\left[\begin{array}{cccc}
1+\frac{5}{3} p & 0 & -\frac{2 \sqrt{5}}{3} p & -\frac{\sqrt{5}}{3} p \\
0 & 1 & 0 & 0 \\
\frac{2 \sqrt{5}}{3} p & 0 & 1-\frac{4}{3} p & -\frac{2}{3} p \\
0 & -\sqrt{5} & 0 & 2 \\
1 & 0 & 0 & 0 \\
0 & 0 & 1 & 0 \\
0 & -2 & 0 & \sqrt{5}
\end{array}\right] \\
& {\left[\begin{array}{ccccc}
3 & 0 & -\frac{2}{3} p & 1-\frac{1}{3} p
\end{array}\right]}
\end{aligned}
$$

and the spectral factor, obtained for instance as the rest of the procedure is now:

$$
\Sigma_{21}=\left[\begin{array}{cc}
-\frac{1}{\sqrt{5}} \frac{p}{p+1} & \frac{2}{p+2} \\
-\frac{1}{\sqrt{5}} \frac{1}{p+1} & 0
\end{array}\right]
$$

We have obtained at the same time an embedding, a cascade synthesis and a spectral factorization.

b) The general case: Again, we restrict ourselves to the physical case whereby the functions considered are all meromorphic. A complete description of a meromorphic $J$-unitary factor is given by Potapov [32], whereby an infinite number of sections of type (III.4.18) and (1II.4.19) may occur (Darlington and Brune sections) and an essential singularity at $\infty$ is represented by a factor of the type:

$$
\Theta^{S}=\int_{\int}^{\frac{l}{p}} d E(t)
$$

whereby $E(t) J$ is a monotone increasing family of Hermitian matrices and $t=\operatorname{tr}[E(t) J]$. Note that a Brune section of type (III.4.19) is a special case of (III.4.20), with $E(t)=u \tilde{u} J h(t)$ ( $h$ being the Heaviside step function). In general $E(t)$ will have a continuous part and a stepfunction part. Approximating $E(t) J$ by means of a step function, we can approximate the integral (III.4.20) by a finite cascade of sections of the following types:

$$
\begin{gathered}
e^{ \pm r p u \tilde{u} J}, \quad r>0, \quad \tilde{u} J u= \pm 1 \\
e^{r p u \tilde{u} J}=1_{2 n}+r p u \tilde{u} J, \quad r>0, \quad \tilde{u} J u=0 .
\end{gathered}
$$

(III.4.21b)

The factor (III.4.21a) is simply the transmission matrix of a uniform transmission line together with some connecting circuitry (ideal transformers or gyrators). Depending on the sign in (III.4.21a) we have either

$$
e^{r p u \bar{u} J}=1_{2 n}-u \tilde{u} J+e^{p} u \tilde{u} J, \quad \tilde{u} J u=1
$$

or

$$
e^{-r p น \tilde{u} J}=1_{2 n}+u \tilde{u} J-e^{-r p} u \tilde{u} J, \quad \tilde{u} J u=-1
$$

so that $\Theta^{S}$ becomes a polynomial matrix in $p, e^{r_{1} p}, \cdots r$, $e^{r} n^{p}$ for some real $r_{i}$. Thus, we can assert that a cascade consisting of a finite number of sections, each either a Darlington, Brune, or transmission line section of type (III.4.21a) realizes a $\Sigma_{21}^{-1}$ and an $S \Sigma_{21}^{-1}$ which are polynomial in $p$ and some $e$ powers. We will call such matrices of finite type. Following (as yet unanswered) questions immediately arise:

1) Suppose $\Sigma_{21}^{-1}$ of finite type, is it true that the corresponding $S \Sigma_{21}^{-1}$ is also of finite type, or else, what are the conditions on $\Sigma_{\mathbf{2 1}}^{-1}$ for this to be the case?

2) Suppose that both $\Sigma_{21}^{-1}$ and $S \Sigma_{21}^{-1}$ are of finite type, is it true that $\Theta^{S}$ is also of finite type? And if so, what is an algorithm to obtain $\Theta^{S}$ out of (III.4.10)?

Part of the problem is that, although $\Sigma_{21}^{-1}$ may be of finite type, it is not true that (III.4.10) or $S$ is of finite type. 
No good, practical criterium of degree reduction of (III.4.10) is available, although some attempts which work in individual cases are shown in [15]. It is possible to generalize the procedure for the rational case to fit this case, but new difficulties arise mainly in connection with the existence of the factors.

\section{Conclusions}

We have represented an analysis of linear distributed systems and networks, using the unifying theory of invariant subspaces and Hardy functions. Thus in Section II, we analyzed state space models and transfer functions and their interelations. We saw that for a fairly large class of systems the wellknown relation between natural modes and poles of lumped system theory generalizes in a natural way. We believe that the theory presented here provides the fundamentals for the development of a detailed frequency domain analysis of distributed systems in Hilbert spaces with potential applications to design (exact or approximate) similar to the one presented in Rosenbrock [1] , [82]. The concept of spectral minimality plays a fundamental role in relating time domain (state space) and frequency domain analysis. We have also indicated how a module theoretic analysis on the lines of Kalman [62] can be generated using the methods presented here. We emphasized the role played by functions which have meromorphic pseudo continuations of bounded type in some left half plane (strictly non cyclic or roomy functions).

In Section III we have analyzed lumped-distributed networks and provided various synthesis methods. In addition we have established the relation between state realizations and frequency domain analysis of scattering matrices. When the scattering matrix is roomy a complete lossless synthesis can be derived using representation formulas of Potapov, at least in principle. In the case of scattering matrices which are not roomy, no lossless embedding exists and hence no generalized Darlington or Oono-Yasuura synthesis. Through coprime factorization of a suitable matrix directly derived from the scattering matrix we obtained spectral factors as well as a lossless embedding in a minimal fashion. This procedure provides a new and very practical algorithm for spectral factorization in the case of rational matrices. We also developed a natural generalization of the degree concept for nonrational matrices.

Throughout the paper we have used coprime factorizations of transfer functions or scattering matrices which are roomy (strictly non cyclic). These factorizations should remind the reader of the factorizations for lumped systems in Rosenbrock [1]. We believe that they will prove to be an important tool in analysis of such diverse problems as quadratic cost problems (implementation of controllers), distributed filtering theory (spectral factorization) and stability of distributed systems and networks [81].

An important direction for further investigation, is the development of practical algorithms for these factorizations and their use in the development of a systematic approximation theory of distributed systems and networks, based on decomposition into subsystems.

\section{REFERENCES}

[1] H. H. Rosenbrock, State-Space and Multivariable Theory. New York: Wiley, 1970 .

[2] J. S. Baras, "Intrinsic models for infinite dimensional linear systems," Ph.D. dissertation, Harvard Univ., Cambridge, MA.,
Sept. 1973.

[3] J. S. Baras and R. W. Brockett, " $\mathrm{H}^{2}$ functions and infinite dimensional realization theory," SIAM J. Contr., vol. 13, no. 1, pp. 221-241, Jan. 1975.

[4] J. S. Baras, R. W. Brockett, and P. A. Fuhrmann, "State space models for infinite dimensional systems," IEEE Trans. A utomat. Contr., Special Issue on Identification and Time Series Analysis, vol. AC-19, pp. 693-700, Dec. 1974

[5] J. S. Baras, "On canonical realizations with unbounded infinitesimal generators," in Proc. 11 th Annu. Allerton Conf. Circuits and System Theory, pp. 1-10, Oct. 1973.

[6] _- "Harmonic analysis of realizations for linear distributed parameter systems," Tech. Rep., Elec. Eng. Dep., Univ. Maryland, College Park, July 1974.

[7] _- "Lumped distributed network synthesis and infinite dimensional realization theory," in Proc. 1974 IEEE Int. Symp. Circuits and Systems, pp. 76-80, Apr. 1974.

[8] —- "Natural models for infinite dimensional systems and their structural properties," in Proc. 8th Annu. Princeton Conf. Inform. Sci. Systems, pp. 195-199, Mar. 1974.

[9] P. Dewilde, "Cascade scattering matrix synthesis," Tech. Rep. Inform. Syst. Lab., Stanford Univ., Stanford, CA, 1970.

[10] - "Roomy scattering matrix synthesis," Tech. Rep., Dep. Mathematics, Univ. California, Berkeley, 1971.

[11] --, "Input-output description of roomy systems," in SIAM J. Control, to be published.

[12] - "Coprime decomposition in roomy system theory," Tech. Rep. Katholieke Univ. Leuven, Leuven, Belgium, 1974.

[13] P. Dewilde, V. Belevitch, and R. W. Newcomb, "On the problem of degree reduction of a scattering matrix by factorization," J. Franklin Inst., vol. 291, no. 5, pp. 387-401, May 1971

[14] P. Dewilde, "On the finite unitary embedding theorem for lossy scattering matrices," in Proc. 1974 European Conf. Circuit Theory and Design (IEE, London).

[15] - "Input-output $\mathrm{L}_{2}$ system theory and scattering matrix synthesis," Tech. Rep., Dep. Elektrotechniek, Katholieke Univ. Leuven, Leuven, Belgium, 1975.

[16] - "On the synthesis of networks using coprime factorization techniques," Tech. Rep., Dep. Elektrotechniek, Katholieke Univ. Leuven, Leuven, Belgium.

[17] P. A. Fuhrmann, "On realization of linear systems and applications to some questions of stability," Math. Syst. Theory, vol. 8, pp. 132-141, 1975 .

[18] - "Exact contollability and observability and realization theory in Hilbert space," J. Math. Anal. Appl., to be published.

[19] -, "Realization theory in Hilbert space for a class of transfer functions,"J. Funct. Anal., vol. 18, pp. 338-349, 1975.

[20] - "On canonical realization of sums and products of nonrational transfer functions," in Proc. 8th Princeton Conf. Information Sci. Syst., pp. 213-217, 1973.

[21] - "On Hankel operator ranges, meromorphic pseudo continuations and factorization of operator valued analytic functions," J. London Math. Soc., to be published.

[22] _-, "Factorization theorems for a class of bounded measurable operator valued functions," to be published.

[23] -, "On spectral minimality of the shift realization," to be published.

[24] J. W. Helton, "Discrete time systems operator models and scattering theory," J. Func. Anal., vol. 16, pp. 15-38, 1974.

[25] R. G. Douglas and J. W. Helton, "Inner dilations of analytic matrix functions and Darlington synthesis," Acta Sci. Math Sz., vol. 34, pp. 301-310, 1973.

[26] H. Helson, Lectures of Invariant Subspaces. New York: Academic Press, 1964.

[27] A. Beurling, "On two problems concerning linear transformations in Hilbert space," Acta Math., vol. 81, pp. 239-255, 1949.

[28] P. D. Lax, "Translation invariant subspaces," Acta. Math., vol. 101, pp. 163-178, 1959.

[29] K. Hoffman, Banach Spaces of Analytic Functions. Englewood Cliffs, NJ: Prentice Hall, 1962.

[30] P. L. Düren, Theory of $H^{p}$ Spaces. New York: Academic Press, 1970.

[31] B. Sz. Nagy and C. Foias, Harmonic Analysis of Operators on Hilbert Space. Amsterdam, The Netherlands: North Holland, 1970.

[32] V. P. Potapov, "The multiplicative structure of J-contractive matrix functions," Amer. Math. Soc., (Transl. Ser. 2), vol. 15, pp. 131-243.

[33] A. V. Efimov, "Realization of reactive J-expanding matrix functions," (in Russian), Akad. Nauk Armjansk, SSSR Izv., vol. V, no. 1 , pp. $54-63,1970$.

[34] I. P. Ginzburg, "On multiplicative representations of J-non expansive operator functions I," Amer. Math. Soc., (Transl. Ser. 2), vol. 96, pp. 189-221, 1970; II: Ibid., pp. 223-254.

[35] V. P. Potapov, "General theorems on the structure and detaching 
of elementary factors of analytical matrix functions," (in Russian), Dokl. Akad. Nauk. Amjansk, SSSR, vol. XLVIII, no. 5, pp. 257-263, 1969.

[36] I. V. Kovalishina and V. P. Potapov, "The multiplicative structure of analytical real J-non contracting matrix functions," (in Russian), Izv. Akad. Nauk Armjanskoii SSSR, vol. XVIII, no. 6, pp. 3-10, 1965.

[37] M. S. Livšic, Operators, Oscillations, Waves, Open Systems, A.M.S. Transl. Math. Monogr., vol. 34, 1973.

[38] S. Bochner and K. Chandrasekharan, Fourier Transforms. Princeton, NJ: Princeton University Press, 1949.

[39] K. Yosida, Functional Analysis. New York: Springer-Verlag, 1965.

[40] H. Wold, A Study in the Analysis of Stationary Time Series, Uppsala, Sweden: Almqvist and Wiksell, 1938 (2nd ed. 1954).

[41] N. Wiener and P. Masani, "The prediction theory of multivariate stochastic processes I. The regularity condition," Acta Math., vol. 98, pp. 111-150, 1957; "II. The linear predictor" Ibid., vol. 99 , pp. 93-137, 1958 .

[42] P. Masani, "Shift invariant spaces and prediction theory," Acta Math., vol. 107, pp. 275-290, 1962.

[43] A. N. Kolmogorov, "Sur l'interpolation et extrapolation de suites stationnaires," C. R. Acad. Sci., vol. 208, p. 2043, 1939.

[44] -, "Stationary sequences in Hilbert space," Bull. Math. Univ. Moscow, vol. 2, no. 6, 1941 (translated by N. Artin).

[45] - "Interpolation and extrapolation of stationary random sequences," Bull Acad. Sci. USSR (Ser. Math.), vol. 5, 1941.

[46] M. G. Krein, "On a generalization of some investigations of G. Szegö, W. M. Smirnov and A. N. Kolmogorov," Dokl. Akad. Nauk SSSR, vol. 46, pp. 91-94, 1945.

[47] -, "On a problem of extrapolation of A. N. Kolmogorov," Dokl. A kad. Nauk SSSR, vol. 46, pp. 306-309, 1945.

[48] J. L. Lions, Optimal Control of Systems Governed by Partial Differential Equations. New York: Springer-Verlag, 1971.

[49] A. G. Butkovskiy, Theory of Optimal Control of Distributed Parameter Systems. New York: American Elsevier, 1969.

[50] D. L. Russell, "Nonharmonic Fourier series in the control theory of distributed parameter systems," J. Math. Anal. Appl., vol. 18, no. 3, pp. 542-559, 1967.

[51] —, "Boundary value control of the higher dimensional wave equation," SIAM J. Control, vol. 9, no. 1, pp. 29-42, 1971.

[52] P. D. Lax and R. S. Phillips, Scattering Theory. New York: Academic Press, 1967.

[53] J. Hale, Functional Differential Equations. New York: SpringerVerlag, 1971.

[54] M. C. Delfour and S. K. Mitter, "Hereditary differential systems with constant delays, I-General Case," J. Diff. Equations, vol. 12, pp. 213-235, 1972.

[55] M. Delfour, "Linear hereditary differential systems and their control," Univ. Montreal, Cent. Rech. Math., Rep. CRM-310, Aug. 1973.

[56] M. Delfour, C. McCalla, and S. K. Mitter, "Stability and the infinite-time quadratic cost problem for linear hereditary differential systems," Univ. Montreal, Cent. Rech. Math., Rep. CRM-273, Apr. 1973.

[57] T. Kato, Perturbation Theory of Linear Operators. New York: Springer-Verlag, 1966

[58] A. V. Balakrishnan, Introduction to Optimization Theory in a Hilbert Space. Berlin, Germany: Springer-Verlag, 1971.

[59] R. W. Brockett, Finite Dimensional Linear Systems. New York: Wiley, 1970

[60] A. V. Balakrishnan, "System theory and stochastic optimization," Proc. NATO Advanced Study Inst. Network and Signal Theory, Bournemouth, England, Sept. 1972.

[61] G. Krein, "Integral equations on a half line with kernels depending on the difference of the arguments," Usp. Mat. Nauk, vol. 13 pp. 3-120, 1958.

[62] R. E. Kalman, P. L. Falb, and M. A. Arbib, Topics in Mathematical System Theory. New York: McGraw-Hill, 1969.

[63] A. Bensoussan and J. P. Aubin, "Models of representation of linear time invariant systems in continuous time," Math. Res. Cent., Univ. Wisconsin, Madison, Rep. MRC 1286, Sept. 1972.

[64] J. S. Baras, "Algebraic structure of infinite dimensional linear systems in Hilbert space," in Proc. CNR-CISM Symp. Advanced School on Algebraic System Theory (Udine, Italy), June 1975.

[65] E. Kamen, "A distributional-module theoretic representation of linear dynamical continuous-time systems," Ph.D. dissertation, Stanford Univ., Stanford, CA, May 1971.

[66] L. A. Gould and M. A. Murray-Lasso, "On the modal control of distributed systems with distributed feedback," IEEE Trans. Automat. Contr., vol. AC-1 1, pp. 729-737, Oct. 1966.

[67] R. G. Douglas, H. S. Shapiro, and A. L. Shields, "Cyclic vectors and invariant subspaces for the backward shift operator," $A n n$. Inst. Fourier (Grenoble, France), vol. 20, 37-76, 1971.

[68] J. W. Moeller, "On the spectra of some translation invariant spaces," J. Math Anal. Appl., vol. 4, pp. 276-296, 1962.

[69] V. Belevitch, Classical Network Theory. San Francisco, CA: Holden Day, 1968.

[70] D. Arov, "Darlington's method for dissipative systems," Sov. Phys. Dok., vol. 16, no. 11, pp. 954-956, May 1972.

(71) V. Belevitch, "Factorization of scattering matrices with applications to passive network synthesis," Phil. Res. Rep., vol. 18, no. 4, pp. 275-317, Aug. 1963.

[72] S. Darlington, "Synthesis of reactance 4-poles which produce prescribed insertion loss characteristics," J. Math Phys., vol. 18, pp. 257-355, Sept. 1939.

[73] Y. Oono and K. Yasuura, "Synthesis of finite passive 2-terminal networks with prescribed scattering matrices," Memo. Fac. Eng., Kyushu Univ., Kyushu, Japan, vol. 14(2), pp. 125-177, May 1954.

[74] R. W. Newcomb, Linear Multiport Synthesis. New York: McGraw-Hill, 1966.

[75] L. A. Zadeh and C. A. Desoer, Linear System Theory. New York: McGraw-Hill, 1963.

[76] W. Rudin, Real and Complex Analysis. New York: McGrawHill, 1966.

[77] T. Koga, "Synthesis of finite passive $n$-ports with prescribed passive real matrices of several variables," IEEE Trans. Circuit Theory, vol. CT-15, Mar. 1968.

[78] T. Koga, "Synthesis of a resistively terminated cascade of uniform transmission lines and lumepd passive lossless two-ports," IEEE Trans. Circuit Theory, vol. CT-18, July 1971.

[79] A. G. Dominguez, "On some canonical factorization formulae for scattering matrices, with applications to circuit synthesis," Tech. Rep., Univ Calif., Berkeley, 1967.

[80] -, "On the factorization of scattering matrices, chain matrices and transfer matrices. Tech. Kep. Dep. Mat., Univ. Buenos Aires, Buenos Aires, Argentina, 1973.

[81] F. M. Callier and C. A. Desoer, " $L P$ stability $(1 \leqslant p \leqslant \infty)$ of multivariable non-linear time varying feedback systems that are open loop unstable,"Int. J. Control, no. 1, pp. 65-72, 1974.

[82] H. H. Rosenbrock, Computer Aided Control System Design. New York: Academic Press, 1974.

[83] R. Saeks, Resolution Space Operators and Systems. Heidelberg, Germany, Springer-Verlag, 1973.

[84] R. Saeks, "The factorization problem-A survey," in Resolution Space, Networks and Non-Self-Adjoint Spectral Theory, Lubbock, TX: Texas Univ. Press, 1975.

[85] R. E. Kalman and M. L. J. Hautus, "Realization for continuoustime linear dynamical systems: Rigorous theory in the style of Schwartz," in Ordinary Differential Equations, (1971, NRLMRC Conf.), New York: Academic Press, 1972. 